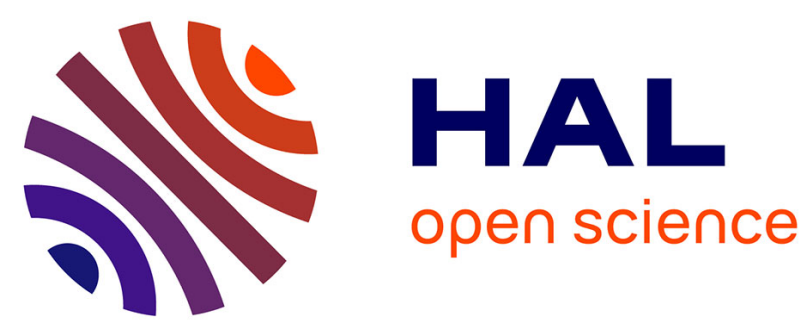

\title{
Selective Suzuki-Miyaura monocouplings with symmetrical dibromoarenes and aryl ditriflates for the one-pot synthesis of unsymmetrical triaryls
}

C. Minard, C. Palacio, K. Cariou, Robert H Dodd

\section{- To cite this version:}

C. Minard, C. Palacio, K. Cariou, Robert H Dodd. Selective Suzuki-Miyaura monocouplings with symmetrical dibromoarenes and aryl ditriflates for the one-pot synthesis of unsymmetrical triaryls. European Journal of Organic Chemistry, 2014, 2014 (14), pp.2942-2955. 10.1002/ejoc.201400090 . hal-00994366

\section{HAL Id: hal-00994366 https://hal.science/hal-00994366}

Submitted on 26 Nov 2020

HAL is a multi-disciplinary open access archive for the deposit and dissemination of scientific research documents, whether they are published or not. The documents may come from teaching and research institutions in France or abroad, or from public or private research centers.
L'archive ouverte pluridisciplinaire HAL, est destinée au dépôt et à la diffusion de documents scientifiques de niveau recherche, publiés ou non, émanant des établissements d'enseignement et de recherche français ou étrangers, des laboratoires publics ou privés. 


\title{
Selective Suzuki-Miyaura Mono-Couplings with Symmetrical Dibromoarenes and Arylditriflates for One-pot Synthesis of Unsymmetrical Triaryls.
}

\author{
Corinne Minard, Carole Palacio, Kevin Cariou, Robert H. Dodd*
}

Keywords: Suzuki-Miyaura, selective coupling, potassium trifluoroborates, triaryls

The various parameters that would permit achievement of selective Suzuki-Miyaura mono-couplings on symmetrical dihaloarenes were studied. Using operationally simple conditions and widely available reagents high selectivity and efficiency can be obtained for a broad range of substrates.
The 38 different examples described provide a valuable toolbox for rapid access to unsymmetrical triaryls, as illustrated by the preparation of diarylpyridine $\mathbf{8}$, terphenyl 9 and diarylpyrrole $\mathbf{1 0}$.
[*] Centre de Recherche de Gif, Institut de Chimie des Substances Naturelles, UPR 2301, CNRS, Avenue de la Terrasse, 91198 Gif-surYvette, France

Email: robert.dodd@cnrs.fr

Supporting information for this article is available on the WWW under http://dx.doi.org/10.1002/ejoc.xxxxxxxxx.

\section{Introduction}

Polyaromatic sequences are widely found in a large variety of organic compounds of both biological and physical interest. As a consequence, many procedures have been developed for their preparation. Metal-catalyzed cross-coupling reactions ${ }^{1}$ and, most prominently, the Suzuki-Miyaura reaction ${ }^{2}$ probably constitute the most efficient strategies to construct these scaffolds through C-C bond formation. However, building an unsymmetrical triaryl requires a certain degree of discrimination between the various reacting sites. The use of two different halides or pseudo-halides allows such a differentiation but often requires tedious stepwise protocols for the preparation of the starting material. ${ }^{3}$ An alternative lies in the synthesis of a substrate bearing identical halides having different steric and/or electronic environments. ${ }^{4} \mathrm{~A}$ simpler approach would be to use symmetrical di-haloarenes, which are more readily available, and to perform a selective mono-coupling in order to ensure the desymmetrization of the starting material. The resulting halodiarene would then constitute a versatile platform for subsequent functionalization. Despite its apparent simplicity, only a handful of studies have been fully devoted to this strategy. In 2005, Uozumi reported that such a monoarylation of dibromoarenes could be achieved using an amphiphilic supported palladium (II) complex and an excess of triphenylphosphine ${ }^{5}$ but Sherburn showed that diarylation was the preferred outcome for diiodoarenes, while monoarylation of dibromoarenes could be achieved when using them in excess. ${ }^{6}$ More recently, $\mathrm{Hu}$ also described that the use of an excess of triphenylphosphine could efficiently favor the formation of monoarylated compounds starting from dibromoarenes (though mainly limited to ortho disubstituted compounds, where steric interactions already tend to favor the formation of a mono-arylated adduct). ${ }^{7}$ McNulty conducted a similar study on dichlorobenzenes, ${ }^{8}$ while the Langer group developed various sets of conditions for selective coupling on polyhalogenated substrates. ${ }^{9}$ In our case, we have previously shown that, since electron-rich arylboronic acids react faster than their electron-poor counterparts, selective monoarylations (with indole boronic acid) ${ }^{10}$ and one-pot simultaneous dicouplings yielding unsymmetrical adducts could be performed. ${ }^{11}$ This latter approach can be extremely valuable for accessing biologically interesting targets such as lamellarin A4 and $\mathrm{G}^{12}$ or ningalin $\mathrm{B}^{13}$ (Figure 1), and their analogs. ${ }^{14}$
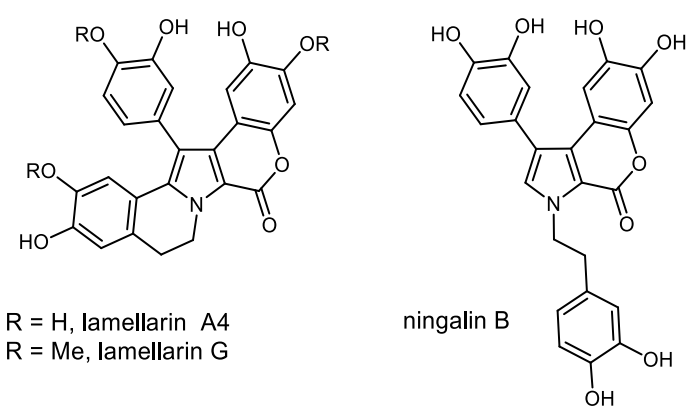

Figure 1. Triaryl Marine Natural Products.

However, our one-pot procedure is somewhat limited by the necessity of having different electron densities for each reactant, while studies by other groups also have some limitations in terms of scope and practicality. ${ }^{5,7}$ Indeed, a widely applicable and general strategy for this purpose is still in need for the synthetic community. In this context, we sought to study the mono-coupling of various symmetrical aryl-dibromides and -ditriflates with a representative panel of boron derivatives in order to develop an efficient and operationally simple protocol that could eventually be amenable to one-pot desymmetrizing-difunctionalization procedures. When looking into the mechanism of the Suzuki-Miyaura cross-coupling it appears that in order to favor the mono-coupling a certain control over the oxidative addition is required. Indeed, oxidative addition of the 14-electron Pd (0) complex to one of the two C-X bonds of dihalo compound 1 would give rise to complex 2 (Scheme 1). After a ligand exchange between $\mathrm{X}$ and a hydroxide, transmetallation with the boronic acid ${ }^{15}$ followed by reductive elimination would then deliver the desired halo-diaryl 3. At this stage, the 14-electron $\operatorname{Pd}(0)$ species is regenerated and can either react with $\mathbf{1}$, which is the desired manifold, or react with $\mathbf{3}$ to generate complex $\mathbf{4}$ and, thereafter, the unwanted symmetrical triaryl $\mathbf{5}$. 


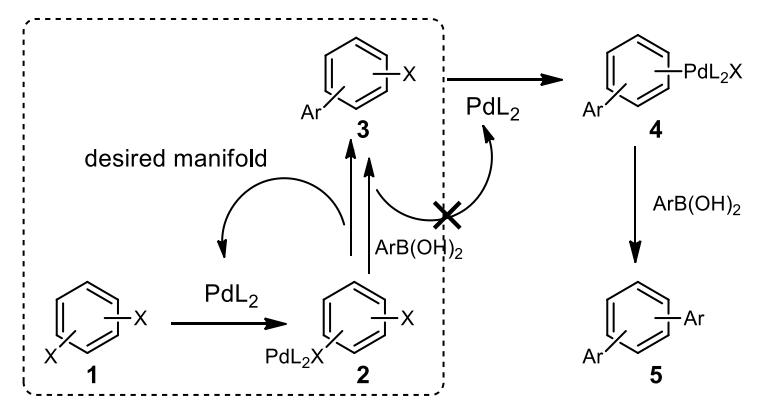

Scheme 1. Mono- vs. di-coupling.

\section{Results and Discussion}

In theory, the initial dihalo compound $\mathbf{1}$ should be slightly more reactive than the resulting mono-haloarene $\mathbf{3}$ toward the oxidative addition and thus any factor that would render this step ratedetermining would increase the chance of a selective mono-coupling pathway. Besides the properties of the dihaloarene, three parameters appeared as potentially critical and relatively flexible: the temperature, the nature of the boron species ${ }^{16}$ and the $\mathrm{Pd} /$ ligand ratio. ${ }^{7}$ As benchmark compounds we started our study by reacting 1 equivalent of 2,6-dibromopyridine 1a with 1 equivalent of $p$ ethoxyphenylboronic acid in a 4/1/2 mixture of toluene/EtOH/ $\mathrm{H}_{2} \mathrm{O}$ in the presence of 8 equivalents of base $\left(\mathrm{Na}_{2} \mathrm{CO}_{3}\right), 50 \mathrm{~mol} \%$ of $\mathrm{KCl}$ and $0.5 \mathrm{~mol} \%$ of $\mathrm{Pd}\left(\mathrm{PPh}_{3}\right)_{4}$ (Table 1, entry 1). ${ }^{17}$ Tetrakis(triphenylphosphine)palladium $(0)$ was chosen because this catalyst is widely available, quite robust and also quite slow in generating the 14electron species. Under these conditions, monoarylated adduct 3aa was the major compound formed but a significant amount of unwanted diarylpyridine 5aa was also observed. Using the corresponding potassium trifluoroborate under the same conditions gave better results in terms of selectivity (entry 2). We then decided to use a slightly higher amount of catalyst but in conjunction with a two-fold excess of triphenylphosphine. Thus, by bringing the Pd:L ratio to $1: 6$ the amount of active 14-electron $\operatorname{Pd}(0)$ complex available would be lower and the oxidative addition into the more reactive dibromopyridine 1a would be favored. ${ }^{18}$ In this case, the boronic acid and the trifluoroborate exhibited identical reactivity and selectivity, but the 87:13 ratio observed was not entirely satisfactory (entries 3,4 ). We then lowered the temperature to rt, which proved detrimental to the reactivity, since full conversion was only obtained after 2 days, but the selectivity was increased to 95/5 (entries 5, 6). When boronic esters (both pinacol and neopentylglycol) were used the selectivity was complete but the conversion extremely low, even after 2 days (entries 7, 8). In order to find a balance between reactivity and selectivity the temperature was raised to $40{ }^{\circ} \mathrm{C}$. Total reaction was now observed after $6 \mathrm{~h}$, except for the boronic esters, and the selectivity remained excellent (entries 9-12) for all compounds. Finally, using only 4 equivalents of base (entries 13-16), both conversion and selectivities remained virtually unchanged, but the best yield of isolated mono-coupled product was obtained when using the trifluoroborate (85\%, entry 14$)$.

Table 1. Mono-coupling Optimization.

\begin{tabular}{|c|c|c|c|c|c|c|c|c|c|}
\hline entry & "B" & $\mathrm{Pd}(\mathrm{mol} \%$ & $\mathrm{PPh}_{3}(\mathrm{~mol} \%)$ & Temp. & $\begin{array}{l}\mathrm{Na}_{2} \mathrm{CO}_{3} \\
\text { (equiv.) }\end{array}$ & t. & Conv. ${ }^{[a]}$ & $\mathbf{3} / \mathbf{5}^{[\mathrm{a}]}$ & yield $^{[b]}$ \\
\hline 1 & $\mathrm{~B}(\mathrm{OH})_{2}$ & 0.5 & 0 & $100^{\circ} \mathrm{C}$ & 8 & $1.5 \mathrm{~h}$ & $100 \%$ & $67 / 33$ & - \\
\hline 2 & $\mathbf{B F}_{3} \mathbf{K}$ & 0.5 & 0 & $100^{\circ} \mathrm{C}$ & 8 & $1.5 \mathrm{~h}$ & $100 \%$ & $90 / 10$ & - \\
\hline 3 & $\mathrm{~B}(\mathrm{OH})_{2}$ & 3 & 6 & $100^{\circ} \mathrm{C}$ & 8 & $2.5 \mathrm{~h}$ & $100 \%$ & $87 / 13$ & - \\
\hline 4 & $\mathrm{BF}_{3} \mathrm{~K}$ & 3 & 6 & $100^{\circ} \mathrm{C}$ & 8 & $2.5 \mathrm{~h}$ & $100 \%$ & $87 / 13$ & - \\
\hline 5 & $\mathrm{~B}(\mathrm{OH})_{2}$ & 3 & 6 & rt & 8 & $2 d$ & $100 \%$ & $95 / 5$ & - \\
\hline 6 & $\mathrm{BF}_{3} \mathrm{~K}$ & 3 & 6 & rt & 8 & $2 \mathrm{~d}$ & $100 \%$ & $95 / 5$ & - \\
\hline 7 & $\mathbf{B}($ pin $)$ & 3 & 6 & rt & 8 & $2 \mathrm{~d}$ & $13 \%$ & $100 / 0$ & - \\
\hline 8 & B(neopent.) & 3 & 6 & rt & 8 & $2 \mathrm{~d}$ & $21 \%$ & $100 / 0$ & - \\
\hline 9 & $\mathrm{~B}(\mathrm{OH})_{2}$ & 3 & 6 & $40^{\circ} \mathrm{C}$ & 8 & $6 \mathrm{~h}$ & $100 \%$ & $91 / 9$ & - \\
\hline 10 & $\mathrm{BF}_{3} \mathrm{~K}$ & 3 & 6 & $40^{\circ} \mathrm{C}$ & 8 & $6 \mathrm{~h}$ & $100 \%$ & $95 / 5$ & - \\
\hline 11 & $\mathrm{~B}($ pin $)$ & 3 & 6 & $40{ }^{\circ} \mathrm{C}$ & 8 & $6 \mathrm{~h}$ & $<50 \%$ & $95 / 5$ & - \\
\hline 12 & B(neopent.) & 3 & 6 & $40{ }^{\circ} \mathrm{C}$ & 8 & $6 \mathrm{~h}$ & $<50 \%$ & $91 / 9$ & - \\
\hline 13 & $\mathrm{~B}(\mathrm{OH})_{2}$ & 3 & 6 & $40^{\circ} \mathrm{C}$ & 4 & $6 \mathrm{~h}$ & $100 \%$ & $91 / 9$ & $70 \%$ \\
\hline 14 & $\mathbf{B F}_{3} \mathbf{K}$ & 3 & 6 & $40^{\circ} \mathrm{C}$ & 4 & $6 \mathrm{~h}$ & $100 \%$ & $95 / 5$ & $85 \%$ \\
\hline 15 & $\mathrm{~B}($ pin $)$ & 3 & 6 & $40^{\circ} \mathrm{C}$ & 4 & $6 \mathrm{~h}$ & $<50 \%$ & $95 / 5$ & $42 \%$ \\
\hline 16 & B(neopent.) & 3 & 6 & $40^{\circ} \mathrm{C}$ & 4 & $6 \mathrm{~h}$ & $<50 \%$ & $95 / 5$ & $51 \%$ \\
\hline
\end{tabular}

[a] Determined by ${ }^{1} \mathrm{H}$ NMR analysis of the crude product mixtures. [b] Isolated yields. 
Here, the nature of the boron function plays an important role as, in our hands, potassium trifluoroborates were found to be more conducive to higher yields. Although their advantages are now well established, ${ }^{19}$ their robustness turns out to be crucial in the case of selective mono-couplings, as using an excess of boron derivative (which could be counterproductive) is not necessary to attain high yields.

Having determined the optimal conditions for this mono-coupling, we next varied the substituents on the trifluoroborate partner. Parasubstituted compounds were first used so that only electronic rather than steric effects would be at play (Table 2, entries 1-4). Substituents with limited electronic effect such as methyl and fluorine reacted smoothly to afford the desired bromo diaryl with $69 \%$ and $70 \%$ yields. On the other hand, electron-withdrawing groups such as nitro and trifluoromethyl gave lower yields, which could not be improved by increasing the temperature in order to compensate for their lower reactivity. It is also important to note that, in the latter case, the difference of reactivity for the oxidative addition step between the starting dibromoarene and the monocoupled product is lowered because of the electron-withdrawing nature of the newly introduced moiety, thus potentially disfavoring a selective process. Meta-methoxyphenyl trifluoroborate reacted poorly at $40{ }^{\circ} \mathrm{C}$ but raising the temperature to $70{ }^{\circ} \mathrm{C}$ and diminishing the reaction time allowed isolation of $62 \%$ of the desired compound (entry 5). The case of ortho-substituted boron reagents was expected to be problematic as steric effects are now at play. Indeed, only modest yields of mono-coupled product were obtained when reacting $o-\mathrm{F}, o-\mathrm{OMe}$ and $o$-OBn phenyltrifluoroborates at $40{ }^{\circ} \mathrm{C}$ (34\%-53\%, entries 6-8).

Table 2. Mono-coupling on 2,6-dibromopyridine.

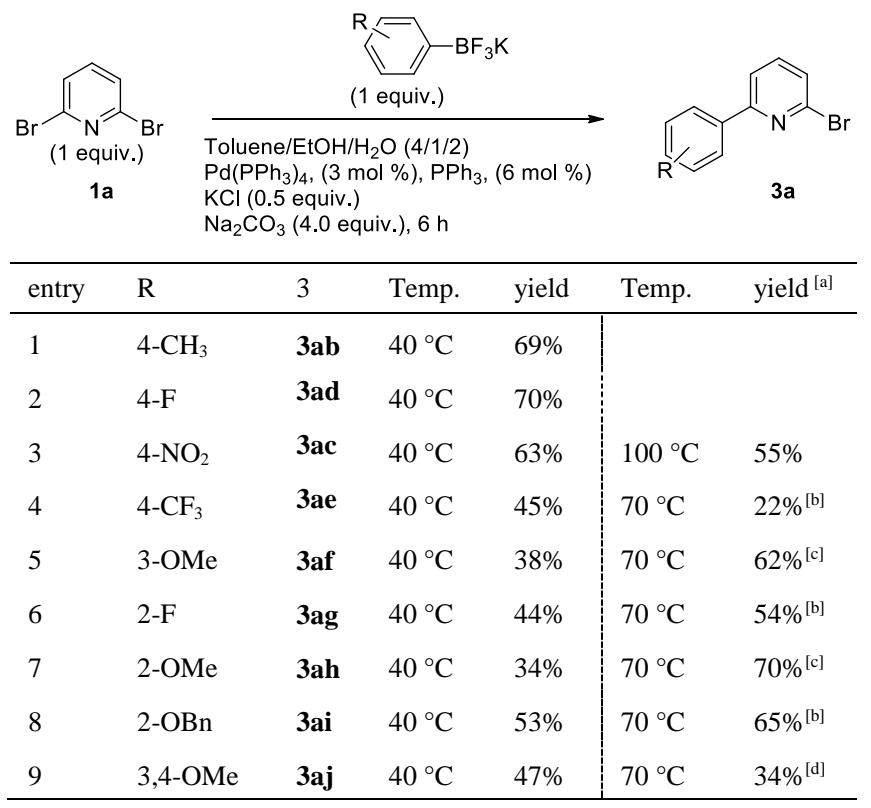

[a] Isolated yields. [b] $4 \mathrm{~h}$ reaction time, $12 \mathrm{~mol} \% \mathrm{PPh}_{3}$. [c] $4 \mathrm{~h}$ reaction time. [d] $4 \mathrm{~h}$ reaction time, a 7:3 ratio for 3aj:5aj was determined by analysis of the crude ${ }^{1} \mathrm{H}$ NMR.

Nevertheless, increasing the temperature to $70{ }^{\circ} \mathrm{C}$ allowed the steric hindrance to be overcome leading to satisfactory yields (54\%$70 \%$ ). In this case, the loss of selectivity that could be expected at a higher reaction temperature was tempered by adding more triphenylphosphine and shortening the reaction time. Finally, 3,4dimethoxyphenyl trifluoroborate was tested (entry 9). While the yield was moderate at $40{ }^{\circ} \mathrm{C}(47 \%)$, increasing the temperature to
$70{ }^{\circ} \mathrm{C}$ only proved detrimental, the main issue being that the high reactivity of this compound tends to favor dicoupling.

The next step was to test these conditions on a wider range of symmetrical dibromo-arenes and -heteroarenes while still varying the electronic properties of the trifluoroborate partner. First, 3,5dibromopyridine $\mathbf{1 b}$ was reacted with 4-OEt-, 4- $\mathrm{CH}_{3}$ - and 4- $\mathrm{NO}_{2}-$ $\mathrm{C}_{6} \mathrm{H}_{4} \mathrm{BF}_{3} \mathrm{~K}$ and the results were quite similar to those obtained with 2,6-dibromopyridine, giving moderate to good yields of the desired bromodiarene (64\%-76\%, Table 3, entries 1-3). When switching to meta- and para-dibromobenzenes (1c, entries 4-6 and 1d, 7-9), the same trend was observed with EDG-substituted borates giving yields above $70 \%$ (entries 4-5,7-8), while EWG-substituted borates led to the desired compound with yields around 50\% (entries 6,9). In these latter cases, a significant amount of di-coupling product was also observed. Because of the limited electronic differentiation between the dibromo starting material and the mono-arylated adduct, the product distribution tends to be roughly statistical (ie a 1:2:1 distribution for 1:3:5, respectively).

Table 3. Mono-coupling on various symmetrical dibromoarenes.

\begin{tabular}{|c|c|c|c|c|c|c|}
\hline $\begin{array}{r}(1 \mathrm{eq} \\
1\end{array}$ & & $\begin{array}{l}\text { Toluene/EtOH/ } / \mathrm{H}_{2} \mathrm{O}(4 \\
\mathrm{Pd}\left(\mathrm{PPh}_{3}\right)_{4},(3 \mathrm{~mol} \%), \\
\mathrm{KCl}(0.5 \text { equiv. }) \\
\mathrm{Na}_{2} \mathrm{CO}_{3} \text { ( } 4.0 \text { equiv.), }\end{array}$ & $\mathrm{Ph}_{3},(6$ & ol \%) & & 3 \\
\hline Entry & & romoarene & $\mathrm{R}$ & 3 & Temp. & $\underset{\text { [a] }}{\text { yield }}$ \\
\hline 1 & & dibromopyridine $\mathbf{1 b}$ & OEt & $3 \mathbf{b a}$ & $40^{\circ} \mathrm{C}$ & $76 \%$ \\
\hline 2 & $1 \mathrm{~b}$ & & $\mathrm{CH}_{3}$ & $3 \mathbf{b b}$ & $40^{\circ} \mathrm{C}$ & $76 \%$ \\
\hline 3 & $1 \mathrm{~b}$ & & $\mathrm{NO}_{2}$ & $3 \mathbf{b c}$ & $40{ }^{\circ} \mathrm{C}$ & $64 \%$ \\
\hline 4 & & dibromobenzene 1c & OEt & $3 \mathbf{c a}$ & $40^{\circ} \mathrm{C}$ & $82 \%$ \\
\hline 5 & $1 \mathrm{c}$ & & $\mathrm{CH}_{3}$ & $3 \mathbf{c b}$ & $40^{\circ} \mathrm{C}$ & $77 \%$ \\
\hline 6 & $1 \mathrm{c}$ & & $\mathrm{NO}_{2}$ & $3 \mathrm{cc}$ & $40^{\circ} \mathrm{C}$ & $47 \%$ \\
\hline 7 & & dibromobenzene 1d & $\mathrm{OEt}$ & 3da & $40^{\circ} \mathrm{C}$ & $76 \%$ \\
\hline 8 & $1 d$ & & $\mathrm{CH}_{3}$ & $3 d \mathbf{d b}$ & $40{ }^{\circ} \mathrm{C}$ & $73 \%$ \\
\hline 9 & 10 & & $\mathrm{NO}_{2}$ & $3 d c$ & $40^{\circ} \mathrm{C}$ & $55 \%$ \\
\hline 10 & & dibromobenzene 1e & OEt & 3ea & $70{ }^{\circ} \mathrm{C}$ & $49 \%$ \\
\hline 11 & 1e & & $\mathrm{CH}_{3}$ & 3eb & $70^{\circ} \mathrm{C}$ & $49 \%[\mathrm{~b}]$ \\
\hline 12 & 1e & & $\mathrm{NO}_{2}$ & 3ec & $70^{\circ} \mathrm{C}$ & $33 \%$ \\
\hline 13 & 1e & & OEt & 3ea & $100^{\circ} \mathrm{C}$ & $74 \%$ \\
\hline 14 & 1e & & $\mathrm{CH}_{3}$ & 3eb & $100^{\circ} \mathrm{C}$ & $40 \%$ \\
\hline 15 & 1e & & $\mathrm{NO}_{2}$ & $3 e c$ & $100^{\circ} \mathrm{C}$ & $49 \%$ \\
\hline 16 & & dibromothiophene 1f & OEt & $3 f a$ & $70^{\circ} \mathrm{C}$ & $53 \%^{[\mathrm{c}]}$ \\
\hline 17 & $\mathbf{1 f}$ & & $\mathrm{CH}_{3}$ & $3 f b$ & $70^{\circ} \mathrm{C}$ & $45 \%^{[\mathrm{c}]}$ \\
\hline 18 & 1f & & $\mathrm{F}$ & $3 f d$ & $70^{\circ} \mathrm{C}$ & $45 \%$ \\
\hline
\end{tabular}

[a] Isolated yields. [b] Conversion as determined by analysis of the ${ }^{1} \mathrm{H}$ NMR: $49 \%$ of $\mathbf{3}, 39 \%$ of $\mathbf{1}$ and $12 \%$ of the dicoupled product. [c] 1.5 equiv. of $\mathrm{ArBF}_{3} \mathrm{~K}$ were used.

For ortho-dibromobenzene 1e the steric interactions, which could favor a selective monocoupling, ${ }^{7}$ mostly proved detrimental as the reaction mixture had to be heated to $70{ }^{\circ} \mathrm{C}$ in order to reach moderate yields (entries 10-12). Increasing the reaction temperature to $100{ }^{\circ} \mathrm{C}$ was necessary to produce yields comparable to those obtained with 
1c and 1d, except in the case of the tolylboronate, for which purification of the desired compound proved challenging (entries 13-15). Finally, 2,5-dibromothiophene 1f was also tested in this reaction and, despite extensive optimization trials, only low to moderate yields could be attained even when an excess of the boron reagent was used (entries 16-18).

In order to further broaden the scope of this study we then turned our attention to various arene ditriflates which, for the most part, can be easily accessed from readily available diphenols and therefore constitute valuable synthons. We first wished to verify which boron function was the most suitable in this case. Running the reaction (using the same operating conditions as for the dibromoarenes) between the ditriflate $\mathbf{6 a}$, derived from hydroquinone, and different para-ethoxybenzeneboron derivatives (Table 4, entries 1-4) clearly showed that the potassium trifluoroborate salt was, again, the optimal choice for a high yielding mono-cross coupling reaction (93\%, entry 2). However, while the $p$-tolyltrifluoroborate gave a satisfactory yield of $69 \%$ (entry 5 ) the para-nitro derivative only led to a complex mixture (entry 6). Resorcinol ditriflate $\mathbf{6 b}$ exhibited a lower reactivity which could readily be enhanced by heating at $70{ }^{\circ} \mathrm{C}$ and prolonging the reaction time to $20 \mathrm{~h}$ to provide the $p$-OEt and $p$ Me products with good yields (entries 7,8).Even the $p-\mathrm{NO}_{2}$ product was obtained with a satisfying $57 \%$ yield under these conditions (entry 9). In the case of the catechol ditriflate $\mathbf{6 c}$ the same trend was observed even at $70{ }^{\circ} \mathrm{C}$ but the yields were lower, probably because of the unfavorable steric interactions (entries 10-12). Finally, pyrrole 6d, which constitutes a key starting material for lamellarin- and ningalin-like derivatives, ${ }^{14}$ was submitted to the same reaction conditions. At $70{ }^{\circ} \mathrm{C}$, excellent yields could be calculated by NMR for both $p$-OEt and $p$-Me compounds, but both products proved quite sensitive to chromatographic purification (entries 13,14). For $p$ - nitrobenzenetrifluoroborate, the desired monocoupled adduct could only be isolated with a modest $31 \%$ yield (entry 15 ). Because of the electron-withdrawing nature of the group introduced, the monocoupled product was quite reactive as evidenced by the isolation of $34 \%$ of the di-coupled adduct, while $27 \%$ of the unreacted starting material was also recovered.

The usefulness of the mono-coupling strategy and the robustness of our conditions were ascertained by accomplishing one-pot desymmetrizing double Suzuki-Miyaura couplings to give the desired triaryl derivatives. ${ }^{20}$ The optimized mono-coupling conditions were first applied to dibromopyridine 1a with $p$ ethoxyphenyl potassium trifluoroborate, only doubling the amount of base introduced, to give arylpyridine 3aa that was not isolated. After the requisite $6 \mathrm{~h}, p$-fluorophenyl potassium trifluoroborate was added and the reaction mixture was heated at $100{ }^{\circ} \mathrm{C}$ for $2 \mathrm{~h}$. This method allowed the isolation of the unsymmetrical diarylpyridine 8 with an excellent $83 \%$ yield (Scheme 2, eq.1). A similar procedure was then applied to 1,3-dibromobenzene 1c (eq. 2) and resorcinol ditriflate $\mathbf{6 b}$ (eq. 3), using $p$-ethoxyphenyl potassium trifluoroborate (under the optimized conditions described in: Table 3-entry 4, for 1c and Table 4-entry 7, for $\mathbf{6 b})$ followed by $p$ trifluoromethylphenylboronic acid. The corresponding terphenyl 9 was obtained in both cases with a satisfactory yield $(58 \%$ and $62 \%$, respectively) considering the lower reactivity of $p$-trifluoromethylsubstituted boron reagents. ${ }^{21}$ Finally, this protocol was also implemented for pyrrole ditriflate 6d using first 3,4dimethoxyphenyl potassium trifluoroborate (under the optimized conditions described in Table 4 entry 13) followed by $o$ benzyloxyphenylboronic acid to give diarylpyrrole $\mathbf{1 0}$ with a $76 \%$ isolated yield (eq. 4).

Table 4. Mono-coupling Optimization on ditriflates.

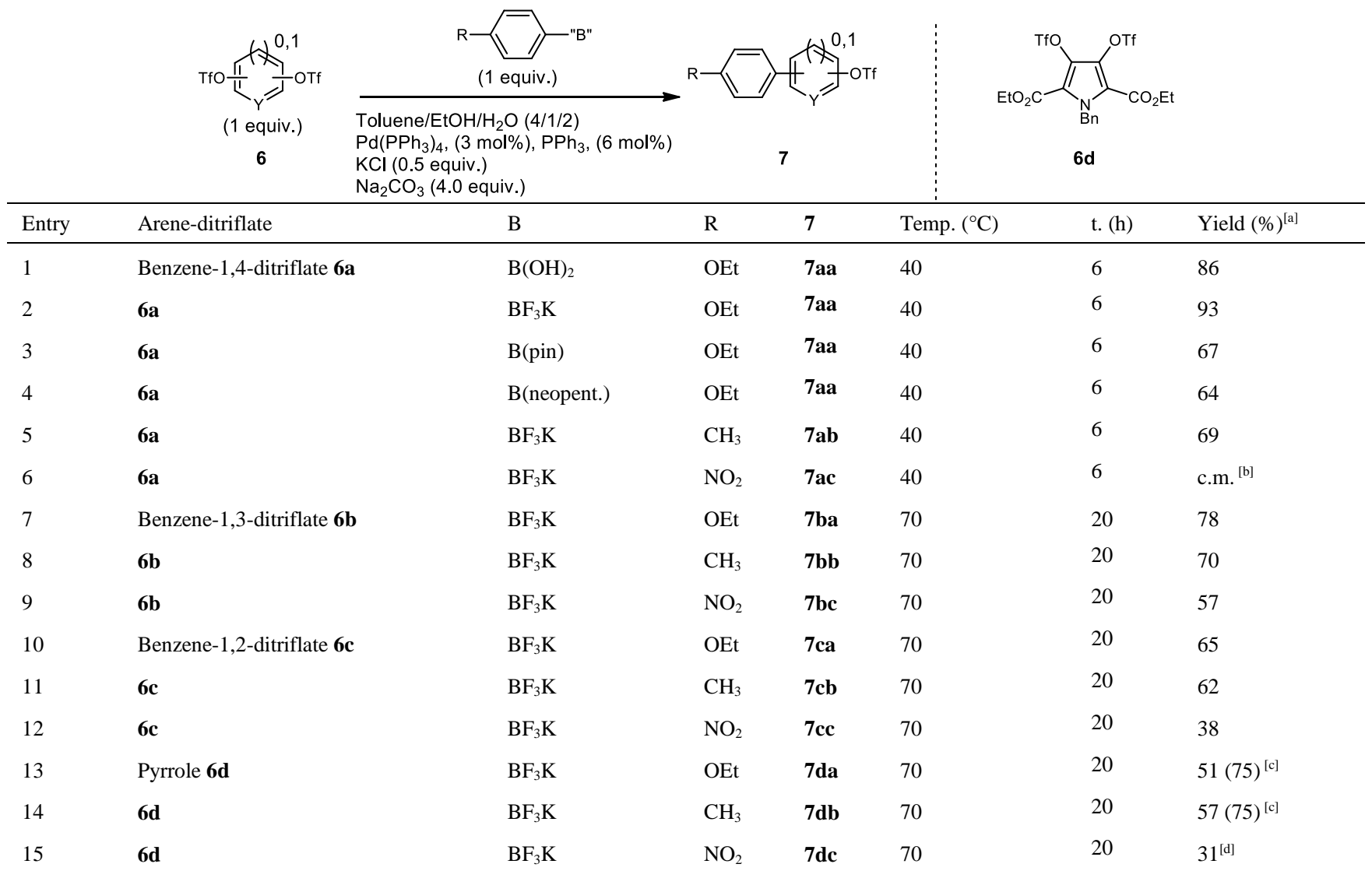

[a] Isolated yields. [b] Complex mixture. [c] Conversion as determined by analysis of the ${ }^{1} \mathrm{H}$ NMR: $75 \%$ of $7,18 \%$ of $\mathbf{6}$ and $7 \%$ of the dicoupled product. [d] $34 \%$ of the symmetrical dicoupled product and $27 \%$ of the starting material were isolated. 

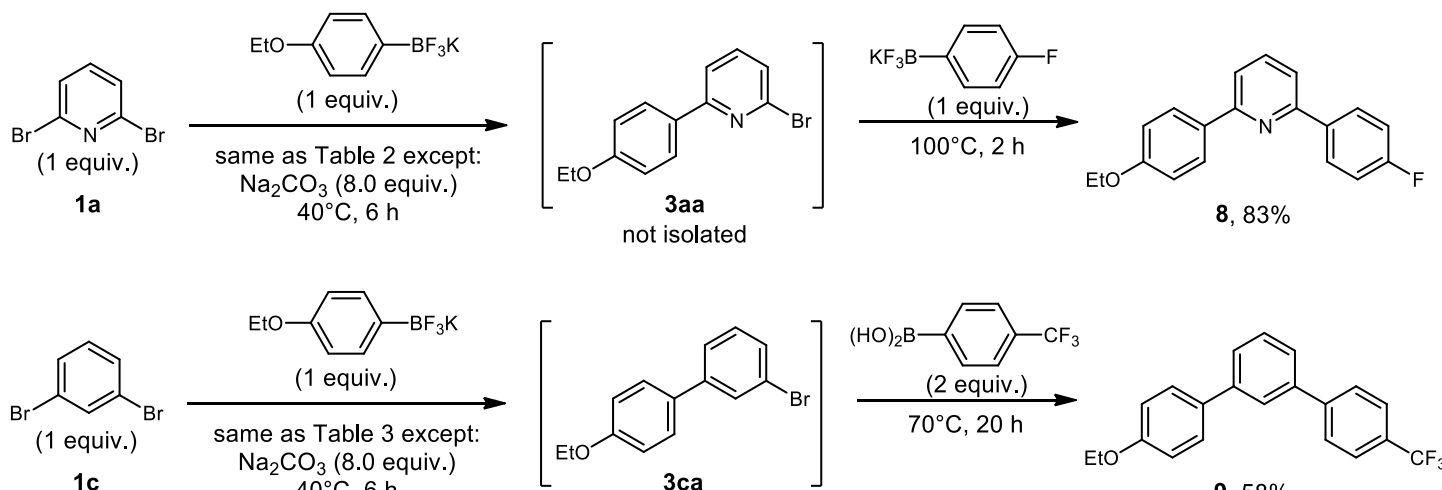

1c $\mathrm{Na}_{2} \mathrm{CO}_{3}$ (8.0 equiv.) $40^{\circ} \mathrm{C}, 6 \mathrm{~h}$<smiles>CCOc1ccc(-c2cccc(Br)c2)cc1</smiles>

not isolated<smiles>CCOc1ccc(-c2cccc(-c3ccc(C(F)(F)F)cc3)c2)cc1</smiles>

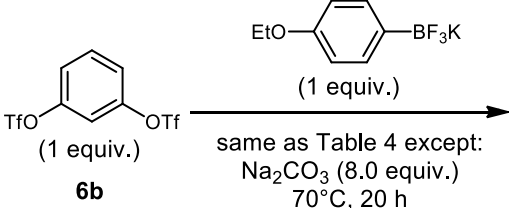

6b $70^{\circ} \mathrm{C}, 20 \mathrm{~h}$<smiles>Cc1ccc(-c2cccc(O)c2)cc1</smiles>

not isolated

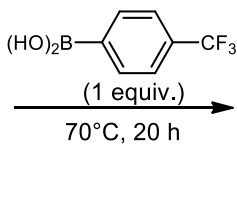<smiles>CCOc1ccc(-c2cccc(-c3ccc(Cl)cc3)c2)cc1</smiles>

9, $62 \%$

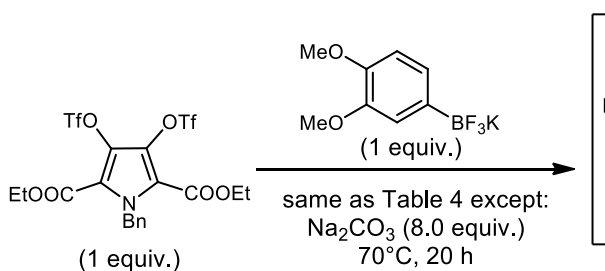

6d<smiles>CCOc1ccc(-c2c(C(=O)OCc3ccccc3)[nH]c(OCC)c2O)cc1O</smiles>

$7 d d$

not isolated

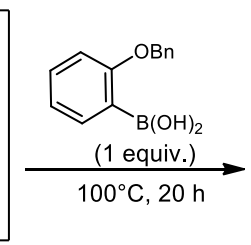<smiles>CCCOc1ccccc1-c1c(C(=O)OCC)[nH]c(C(=O)OCC)c1-c1ccc(OC)c(OC)c1</smiles>

$10,76 \%$

Scheme 2. One-pot synthesis of unsymmetrical triaryls.

This latter example is remarkable because it paves the way for the rapid construction of libraries of ningalin $\mathrm{B}$ analogs from a common platform, and also because isolation of the unstable intermediate mono-triflate is avoided, thus allowing the target compound to be obtained with an excellent yield.

\section{Conclusions}

Overall, we have shown that selective mono Suzuki-Miyaura cross-coupling can be accomplished on a wide range of symmetrical dihaloarenes using operationally simple conditions. By adjusting several parameters such as the catalyst/ligand ratio and the temperature, the desired adducts can be obtained in good yields, without requiring elaborate ligands and/or catalyst systems or the use of an excess of the dihalo-compound. A key feature that emerged from this study is that the most suitable substrates for selective coupling are the EDG-bearing arylboron derivatives. Indeed the EDG has the doubly beneficial role of enhancing the first coupling, which can in turn be run at a lower temperature, and of increasing the difference in reactivity between the dihaloarene and the resulting halodiaryl. This has an impact on the order of the couplings if one wishes to introduce an EDG and an EWG on the same framework. The moiety bearing the electron-withdrawing substituent should thus be incorporated in a second coupling, as the lower reactivity of these compounds can then be compensated by using harsher conditions. Finally, while this selective mono-coupling can grant access to many valuable synthons for further transformations, it also offers opportunities for a second Suzuki-Miyaura cross-coupling to rapidly access triaryl scaffolds. This was showcased by performing highly efficient desymmetrizing one-pot double Suzuki-Miyaura reactions on dibromopyridine $\mathbf{1 a}, 1,3$-dibromobenzene $\mathbf{1 c}$, resorcinol ditriflate $\mathbf{6 b}$ and pyrroleditriflate $\mathbf{6 d}$. Further efforts in this direction, including the preparation and evaluation of libraries of biologically relevant compounds, are currently underway in our laboratory and will be reported in due course.

\section{Experimental Section}

General Methods: Melting points were measured in capillary tubes on a Büchi B-540 apparatus and are uncorrected. Infrared spectra were recorded on a Perkin Elmer Spectrum BX FT-IR spectrometer. Proton $\left({ }^{1} \mathrm{H}\right)$ and carbon $\left({ }^{13} \mathrm{C}\right) \mathrm{NMR}$ spectra were recorded on Bruker spectrometers: Avance 300 $\mathrm{MHz}$ (QNP $-{ }^{13} \mathrm{C},{ }^{31} \mathrm{P},{ }^{19} \mathrm{~F}$ - probe or Dual ${ }^{13} \mathrm{C}$ probe) and Avance $500 \mathrm{MHz}$ (BB0 - ATM probe or BBI - ATM probe). Carbon NMR $\left({ }^{13} \mathrm{C}\right)$ spectra were recorded at 125 or $75 \mathrm{MHz}$, using a broadband decoupled mode with the multiplicities obtained using a JMOD or DEPT sequence. NMR experiments were carried out in deuterochloroform $\left(\mathrm{CDCl}_{3}\right)$, chemical shifts $(\delta)$ are reported in parts per million (ppm) with reference to $\mathrm{CDCl}_{3}\left({ }^{1} \mathrm{H}: 7.24 ;{ }^{13} \mathrm{C}\right.$ : 77.23). The following abbreviations are used for the proton spectra multiplicities: s: singlet, bs: broad singlet, d: doublet, t: triplet, q: quartet, hept: heptuplet, m: multiplet, br: broad. Coupling constants $(J)$ are reported in Hertz (Hz). Mass spectra were obtained either with a LCT (Micromass) instrument using electrospray ionization (ES), or from a Time of Flight 
analyzer (ESI-MS) for the high resolution mass spectra (HRMS). APPI spectra were obtained with an Agilent 1290 Infinity SFC instrument using a Q-ToF 6540 analyser and APPI ionization. Analytical thin-layer chromatography was performed on silica gel $60 \mathrm{~F}_{254}$ on aluminum plates (Merck) and visualized under a UVP Mineralight UVLS-28 lamp (254 nm) and with ninhydrin and $p$-anisaldehyde stains. Preparative thin-layer chromatography was performed on silica gel $60 \mathrm{~F}_{254}$ (Merck, $0.5 \mathrm{~mm}$ ). Flash chromatography was conducted on Merck silica gel $60(40-63 \mu \mathrm{m})$ at medium pressure (300 mbar) or on CombiFlash apparatus (Serlabo Technologies), using standard settings. For Suzuki-Miyaura couplings, solvents were always freshly degassed with argon. Organic extracts were dried over magnesium sulfate $\left(\mathrm{MgSO}_{4}\right)$. All reagents were obtained from commercial suppliers unless otherwise stated. All boronic acids were obtained from Frontier Scientific and other boronic compounds (potassium organotrifluoroborates ${ }^{19}$ and boronic esters ${ }^{22}$ ) were prepared following literature procedures. Pyrrole derivatives were also synthesized according to literature procedures. ${ }^{14 a}$

General Procedure A, Suzuki-Miyaura Monocouplings: The aryldibromide (or arylditriflate, 1.0 equiv.), the boron derivative (1.0 equiv.), sodium carbonate (4.0 equiv.), potassium chloride ( 0.5 equiv.) and triphenylphosphine $(6 \mathrm{~mol} \%)$ were successively introduced in a sealing tube. The tube was capped with a rubber septum and placed under an argon atmosphere. Tetrakis(triphenylphosphine)palladium(0) (3 mol \%) was then added and a mixture of freshly degassed toluene/ethanol/water (4/1/2, v/v/v, respectively) was injected via syringe. The septum was replaced by a screwcap, the tube was sealed and the reaction mixture was stirred at the appropriate temperature $\left(40^{\circ} \mathrm{C}-100^{\circ} \mathrm{C}\right)$ for $6-20 \mathrm{~h}$. The resulting mixture was then cooled to RT, extracted with ethyl acetate and washed with water. The combined organic layers were dried over $\mathrm{MgSO}_{4}$ and concentrated under reduced pressure. Purification of the resulting crude material was performed by flash silica gel chromatography or preparative TLC using the appropriate solvent system.

General Procedure B, Suzuki-Miyaura One-pot Double Couplings: The aryldibromide (or arylditriflate, 1 equiv.), the first boron derivative (1 equiv.) sodium carbonate ( 8 equiv.), potassium chloride ( 0.5 equiv.) and triphenylphosphine ( $6 \mathrm{~mol} \%$ ) were successively introduced in a sealing tube. The tube was capped with a rubber septum and placed under an argon atmosphere. Tetrakis(triphenylphosphine)palladium( $(0)$ ( 3 mol\%) was then added and a mixture of freshly degassed toluene/ethanol/water (4/1/2, v/v/v, respectively) was injected via syringe. The septum was replaced by a screwcap, the tube was sealed and the reaction mixture was stirred at the appropriate temperature $\left(40{ }^{\circ} \mathrm{C}-100{ }^{\circ} \mathrm{C}\right)$ for $6-20 \mathrm{~h}$. The mixture was then cooled until reflux stopped and the second boron derivative (1 equiv.) was then added. Again, the tube was sealed and the reaction mixture was stirred at the appropriate temperature $\left(40{ }^{\circ} \mathrm{C}-100{ }^{\circ} \mathrm{C}\right)$ for an additional $6-20 \mathrm{~h}$. The resulting mixture was then cooled to rt, extracted with ethyl acetate and washed with water. The combined organic layers were dried over $\mathrm{MgSO}_{4}$ and concentrated under reduced pressure. Purification of the resulting crude material was performed by flash silica gel chromatography or preparative TLC using the appropriate solvent system.

2-Bromo-6-(4-ethoxyphenyl)pyridine (3aa): Prepared according to general procedure A by reacting 2,6-dibromopyridine ( $46 \mathrm{mg}, 0.2 \mathrm{mmol})$, with potassium 4-ethoxyphenyltrifluoroborate ( $46 \mathrm{mg}, 0.2 \mathrm{mmol}$ ), sodium carbonate $(85 \mathrm{mg}, 0.8 \mathrm{mmol})$, potassium chloride $(7 \mathrm{mg}, 0.1 \mathrm{mmol})$, triphenylphosphine $\quad(3.0 \quad \mathrm{mg}, \quad 6 \quad \mathrm{~mol} \quad \%) \quad$ and tetrakis(triphenylphosphine)palladium( $(0)(7.0 \mathrm{mg}, 3 \mathrm{~mol} \%)$ in a mixture of degassed toluene/ethanol $(1.40 / 0.35 \mathrm{~mL})$ and degassed water $(0.70 \mathrm{~mL})$. The reaction was stirred at $40{ }^{\circ} \mathrm{C}$ for 6 hours. After extraction, purification of the crude material by flash chromatography on silica gel ( $0 \%$ to $20 \%$ EtOAc in heptane) afforded 3aa as a white powder $(46 \mathrm{mg}, 85 \%) .{ }^{1} \mathrm{H} \mathrm{NMR}(300 \mathrm{MHz}$, $\left.\mathrm{CDCl}_{3}\right): \delta=1.42(\mathrm{t}, J=6.9 \mathrm{~Hz}, 3 \mathrm{H}), 4.06(\mathrm{q}, J=6.9 \mathrm{~Hz}, 2 \mathrm{H}), 6.94(\mathrm{~d}, J=$ $8.8 \mathrm{~Hz}, 2 \mathrm{H}), 7.31(\mathrm{dd}, J=7.6,1.0 \mathrm{~Hz}, 1 \mathrm{H}), 7.51(\mathrm{dd}, J=7.8,7.6 \mathrm{~Hz}, 1 \mathrm{H})$, $7.58(\mathrm{dd}, J=7.8,1.0 \mathrm{~Hz}, 1 \mathrm{H}), 7.91(\mathrm{~d}, J=8.8 \mathrm{~Hz}, 2 \mathrm{H}) ;{ }^{13} \mathrm{C} \mathrm{NMR}(75 \mathrm{MHz}$,
$\left.\mathrm{CDCl}_{3}\right): \delta=15.0\left(\mathrm{CH}_{3}\right), 63.8\left(\mathrm{CH}_{2}\right), 114.8(2 \mathrm{CH}), 118.3(\mathrm{CH}), 125.6(\mathrm{CH})$, $128.5(2 \mathrm{CH}), 130.2(\mathrm{C}), 139.1(\mathrm{CH}), 142.2(\mathrm{C}), 158.5(\mathrm{C}), 160.5(\mathrm{C})$; IR (neat): $v=2975,2926,1606,1574,1547,1512,1432,1421,1392,1251$, 1115, 1042, $790 \mathrm{~cm}^{-1}$; HRMS (ESI): calc. for $\mathrm{C}_{13} \mathrm{H}_{13}{ }^{79} \mathrm{BrNO}_{2}[\mathrm{M}+\mathrm{H}] \mathrm{m} / \mathrm{z}$ 278.0102, found $\mathrm{m} / \mathrm{z} 278.0173$, calc. for $\mathrm{C}_{13} \mathrm{H}_{13}{ }^{81} \mathrm{BrNO}_{2}[\mathrm{M}+\mathrm{H}] \mathrm{m} / \mathrm{z}$ 280.0082 , found $\mathrm{m} / \mathrm{z} 280.0151$.

2-Bromo-6-( $p$-tolyl)pyridine (3ab): Prepared according to general procedure A by reacting 2,6-dibromopyridine ( $46 \mathrm{mg}, 0.2 \mathrm{mmol}$ ), with potassium 4-methylphenyltrifluoroborate $(40 \mathrm{mg}, 0.2 \mathrm{mmol})$, sodium carbonate $(85 \mathrm{mg}, 0.8 \mathrm{mmol})$, potassium chloride $(7 \mathrm{mg}, 0.1 \mathrm{mmol})$, triphenylphosphine $\quad(3.0 \quad \mathrm{mg}, \quad 6 \quad \mathrm{~mol} \%) \quad$ and tetrakis(triphenylphosphine)palladium(0) $(7.0 \mathrm{mg}, 3 \mathrm{~mol} \%)$ in a mixture of degassed toluene/ethanol $(1.40 / 0.35 \mathrm{~mL})$ and degassed water $(0.70 \mathrm{~mL})$. The reaction was stirred at $40{ }^{\circ} \mathrm{C}$ for 6 hours. After extraction, purification of the crude material by flash chromatography on silica gel (0\% to $40 \% \mathrm{CH}_{2} \mathrm{Cl}_{2}$ in heptane) afforded 3ab as a white powder (34 mg, 69\%). ${ }^{1} \mathrm{H}$ NMR (300 MHz, $\left.\mathrm{CDCl}_{3}\right): \delta=2.39(\mathrm{~s}, 3 \mathrm{H}), 7.25(\mathrm{~d}, J=8.2 \mathrm{~Hz}, 2 \mathrm{H}), 7.35(\mathrm{dd}, J=7.6,0.9 \mathrm{~Hz}$, $1 \mathrm{H}), 7.54(\mathrm{dd}, J=7.7,7.6 \mathrm{~Hz}, 1 \mathrm{H}), 7.63(\mathrm{dd}, J=7.7,0.9 \mathrm{~Hz}, 1 \mathrm{H}), 7.87$ (d, $J$ $=8.2 \mathrm{~Hz}, 2 \mathrm{H}) ;{ }^{13} \mathrm{C} \mathrm{NMR}\left(75 \mathrm{MHz}, \mathrm{CDCl}_{3}\right): \delta=21.5\left(\mathrm{CH}_{3}\right), 118.1(\mathrm{CH})$, $126.1(\mathrm{CH}), 127.1(2 \mathrm{CH}), 129.7(2 \mathrm{CH}), 135.1(\mathrm{C}), 139.1(\mathrm{CH}), 140.0(\mathrm{C})$, 142.3 (C), 158.8 (C); IR (neat): $v=3028,2913,2855,1579,1544,1512$, $1429,1413,1124,790 \mathrm{~cm}^{-1}$; HRMS (ESI): calc. for $\mathrm{C}_{12} \mathrm{H}_{11}{ }^{79} \mathrm{BrN}[\mathrm{M}+\mathrm{H}] \mathrm{m} / \mathrm{z}$ 247.9997, found $\mathrm{m} / \mathrm{z} 248.0081$, calc. for $\mathrm{C}_{12} \mathrm{H}_{11}{ }^{81} \mathrm{BrN}[\mathrm{M}+\mathrm{H}] \mathrm{m} / \mathrm{z} 249.9976$, found $\mathrm{m} / \mathrm{z} 250.0058$.

2-Bromo-6-(4-nitrophenyl)pyridine (3ac): Prepared according to general procedure A by reacting 2,6-dibromopyridine ( $46 \mathrm{mg}, 0.2 \mathrm{mmol})$, with potassium 4-nitrophenyltrifluoroborate $(46 \mathrm{mg}, 0.2 \mathrm{mmol})$, sodium carbonate $(85 \mathrm{mg}, 0.8 \mathrm{mmol})$, potassium chloride $(7 \mathrm{mg}, 0.1 \mathrm{mmol})$, triphenylphosphine $\quad(3.0 \quad \mathrm{mg}, \quad 6 \quad \mathrm{~mol} \%) \quad$ and tetrakis(triphenylphosphine)palladium( 0$)(7.0 \mathrm{mg}, 3 \mathrm{~mol} \%)$ in a mixture of degassed toluene/ethanol $(1.40 / 0.35 \mathrm{~mL})$ and degassed water $(0.70 \mathrm{~mL})$. The reaction was stirred at $40^{\circ} \mathrm{C}$ for 6 hours. After extraction, purification of the crude material by flash chromatography on silica gel ( $0 \%$ to $20 \%$ EtOAc in heptane) afforded 3ac as a yellow powder (35 mg, 63\%). ${ }^{1} \mathrm{H}$ NMR (300 MHz, $\left.\mathrm{CDCl}_{3}\right): \delta=7.50(\mathrm{dd}, J=7.9,0.9 \mathrm{~Hz}, 1 \mathrm{H}), 7.66(\mathrm{dd}, J=7.7,7.9 \mathrm{~Hz}, 1 \mathrm{H})$, $7.75(\mathrm{dd}, J=7.7,0.9 \mathrm{~Hz}, 1 \mathrm{H}), 8.15(\mathrm{~d}, J=8.8 \mathrm{~Hz}, 2 \mathrm{H}), 8.29(\mathrm{~d}, J=8.8 \mathrm{~Hz}$, $2 \mathrm{H}) ;{ }^{13} \mathrm{C} \mathrm{NMR}\left(75 \mathrm{MHz}, \mathrm{CDCl}_{3}\right): \delta=120.0(\mathrm{CH}), 124.2(2 \mathrm{CH}), 128.0(2 \mathrm{CH})$, $128.2(\mathrm{CH}), 139.5(\mathrm{CH}), 142.8(\mathrm{C}), 143.6(\mathrm{C}), 148.7(\mathrm{C}), 156.0(\mathrm{C})$; IR (neat): $v=3110,3085,2962,1507,1343,1259,1087,1047,1012,790 \mathrm{~cm}^{-1}$; HRMS (ESI): calc. for $\mathrm{C}_{11} \mathrm{H}_{8}{ }^{79} \mathrm{BrN}_{2} \mathrm{O}_{2}[\mathrm{M}+\mathrm{H}] \mathrm{m} / \mathrm{z}$ 278.9691, found $\mathrm{m} / \mathrm{z}$ 278.9787, calc. for $\mathrm{C}_{11} \mathrm{H}_{8}{ }^{81} \mathrm{BrN}_{2} \mathrm{O}_{2}[\mathrm{M}+\mathrm{H}] \mathrm{m} / \mathrm{z}$ 280.9670, found $\mathrm{m} / \mathrm{z}$ 280.9781 .

2-Bromo-6-(4-fluorophenyl)pyridine (3ad): Prepared according to general procedure A by reacting 2,6-dibromopyridine ( $46 \mathrm{mg}, 0.2 \mathrm{mmol}$ ), with potassium 4-fluorophenyltrifluoroborate $(40 \mathrm{mg}, 0.2 \mathrm{mmol})$, sodium carbonate $(85 \mathrm{mg}, 0.8 \mathrm{mmol})$, potassium chloride $(7 \mathrm{mg}, 0.1 \mathrm{mmol})$, triphenylphosphine $\quad(3.0 \quad \mathrm{mg}, \quad 6.0 \quad \mathrm{~mol} \%) \quad$ and tetrakis(triphenylphosphine)palladium $(0)(7.0 \mathrm{mg}, 3.0 \mathrm{~mol} \%)$ in a mixture of degassed toluene/ethanol $(1.40 / 0.35 \mathrm{~mL})$ and degassed water $(0.70 \mathrm{~mL})$. The reaction was stirred at $40{ }^{\circ} \mathrm{C}$ for 6 hours. After extraction, purification of the crude material using preparative TLC $\left(20 \% \mathrm{CH}_{2} \mathrm{Cl}_{2}\right.$ in heptane) afforded 3ad as a white powder (35 mg, 70\%). ${ }^{1} \mathrm{H}$ NMR (300 MHz, $\left.\mathrm{CDCl}_{3}\right)$ : $\delta=7.12(\mathrm{t}, J=8.8 \mathrm{~Hz}, 2 \mathrm{H}), 7.38(\mathrm{dd}, J=7.5,1.1 \mathrm{~Hz}, 1 \mathrm{H}), 7.56(\mathrm{t}, J=7.5$ $\mathrm{Hz}, 1 \mathrm{H}), 7.61(\mathrm{dd}, J=7.5,1.1 \mathrm{~Hz}, 1 \mathrm{H}), 7.96(\mathrm{dd}, J=8.8,5.2 \mathrm{~Hz}, 2 \mathrm{H}) ;{ }^{13} \mathrm{C}$ $\operatorname{NMR}\left(75 \mathrm{MHz}, \mathrm{CDCl}_{3}\right): \delta=116.0(\mathrm{~d}, J=21.8 \mathrm{~Hz}, 2 \mathrm{CH}), 118.8(\mathrm{CH}), 126.5$ (CH), 129.1 (d, $J=8.7 \mathrm{~Hz}, 2 \mathrm{CH}), 134.0(\mathrm{~d}, J=2.9 \mathrm{~Hz}, \mathrm{C}), 139.3(\mathrm{CH}), 142.4$ (C), 157.7 (C), 164.1 (d, $J=247.6 \mathrm{~Hz}, \mathrm{C}$ ); IR (neat): $v=3079,2924,1596$, 1578, 1547, 1508, 1428, 1414, 1386, 1227, 1124, 851, $790 \mathrm{~cm}^{-1}$; HRMS (ESI): calc. for $\mathrm{C}_{11} \mathrm{H}_{8}{ }^{79} \mathrm{BrFN}[\mathrm{M}+\mathrm{H}] \mathrm{m} / \mathrm{z} 251.9746$, found $\mathrm{m} / \mathrm{z} 251.9834$, calc. for $\mathrm{C}_{11} \mathrm{H}_{8}{ }^{81} \mathrm{BrFN}[\mathrm{M}+\mathrm{H}] \mathrm{m} / \mathrm{z} 253.9725$, found $\mathrm{m} / \mathrm{z} 253.9808$.

2-Bromo-6-(4-(trifluoromethyl)phenyl)pyridine (3ae): Prepared according to general procedure A by reacting 2,6-dibromopyridine (46 mg, $0.2 \mathrm{mmol}$ ), with potassium 4-trifluoromethanephenyltrifluoroborate $(50 \mathrm{mg}$, 
$0.2 \mathrm{mmol}$ ), sodium carbonate ( $85 \mathrm{mg}, 0.8 \mathrm{mmol})$, potassium chloride $(7 \mathrm{mg}$, $0.1 \mathrm{mmol})$, triphenylphosphine $\left(\begin{array}{lllll}3.0 & \mathrm{mg}, & 6 & \mathrm{~mol} & \%\end{array}\right)$ and tetrakis(triphenylphosphine)-palladium( $(0)(7.0 \mathrm{mg}, 3 \mathrm{~mol} \%)$ in a mixture of degassed toluene/ethanol $(1.40 / 0.35 \mathrm{~mL})$ and degassed water $(0.70 \mathrm{~mL})$. The reaction was stirred at $40{ }^{\circ} \mathrm{C}$ for 6 hours. After extraction, purification of the crude material using preparative TLC (10\% EtOAc in heptane) afforded 3ae as a white powder $(27 \mathrm{mg}, 45 \%){ }^{1} \mathrm{H} \mathrm{NMR}\left(300 \mathrm{MHz}, \mathrm{CDCl}_{3}\right): \delta=7.46(\mathrm{dd}$, $J=7.7,1.0 \mathrm{~Hz}, 1 \mathrm{H}), 7.62(\mathrm{t}, J=7.7 \mathrm{~Hz}, 1 \mathrm{H}), 7.67-7.73(\mathrm{~m}, 3 \mathrm{H}), 8.09(\mathrm{~d}, J=$ $8.0 \mathrm{~Hz}, 2 \mathrm{H}) ;{ }^{13} \mathrm{C} \mathrm{NMR}\left(75 \mathrm{MHz}, \mathrm{CDCl}_{3}\right): \delta=119.6(\mathrm{CH}), 124.5(\mathrm{q}, J=270.5$ $\left.\mathrm{Hz}, \mathrm{CF}_{3}\right), 126.0(\mathrm{q}, J=3.8 \mathrm{~Hz}, 2 \mathrm{CH}), 127.5(2 \mathrm{CH}), 127.6(\mathrm{CH}), 132.2(\mathrm{q}, J$ $=47.5 \mathrm{~Hz}, \mathrm{C}), 138.3(\mathrm{C}), 139.4(\mathrm{CH}), 141.1(\mathrm{C}), 142.7$ (C); IR (neat): $v=$ 3079, 2927, 1575, 1551, 1432, 1312, 1110, 1072, 848, 793, $747 \mathrm{~cm}^{-1}$; HRMS (ESI): calc. for $\mathrm{C}_{12} \mathrm{H}_{8}{ }^{79} \mathrm{BrF}_{3} \mathrm{~N}[\mathrm{M}+\mathrm{H}] \mathrm{m} / \mathrm{z}$ 301.9714, found $\mathrm{m} / \mathrm{z}$ 301.9814, calc. for $\mathrm{C}_{12} \mathrm{H}_{8}{ }^{81} \mathrm{BrF}_{3} \mathrm{~N}[\mathrm{M}+\mathrm{H}] \mathrm{m} / \mathrm{z}$ 303.9693, found $\mathrm{m} / \mathrm{z} 303.9799$.

2-Bromo-6-(3-methoxyphenyl)pyridine (3af): Prepared according to general procedure A by reacting 2,6-dibromopyridine ( $46 \mathrm{mg}, 0.2 \mathrm{mmol})$, with potassium 3-methoxyphenyltrifluoroborate ( $43 \mathrm{mg}, 0.2 \mathrm{mmol})$, sodium carbonate $(85 \mathrm{mg}, 0.8 \mathrm{mmol})$, potassium chloride $(7 \mathrm{mg}, 0.1 \mathrm{mmol})$, triphenylphosphine $\quad(3.0 \quad \mathrm{mg}, \quad 6 \quad \mathrm{~mol} \%) \quad$ and tetrakis(triphenylphosphine)palladium( $(0)(7.0 \mathrm{mg}, 3 \mathrm{~mol} \%)$ in a mixture of degassed toluene/ethanol $(1.40 / 0.35 \mathrm{~mL})$ and degassed water $(0.70 \mathrm{~mL})$. The reaction was stirred at $70{ }^{\circ} \mathrm{C}$ for 4 hours. After extraction, purification of the crude material using preparative TLC (30\% $\mathrm{CH}_{2} \mathrm{Cl}_{2}$ in heptane) afforded $3 \mathbf{a f}$ as a white powder $(32 \mathrm{mg}, 62 \%) .{ }^{1} \mathrm{H} \mathrm{NMR}\left(300 \mathrm{MHz}, \mathrm{CDCl}_{3}\right): \delta=3.87(\mathrm{~s}$, $3 \mathrm{H}), 6.93-6.99(\mathrm{~m}, 1 \mathrm{H}), 7.34(\mathrm{~d}, J=7.7 \mathrm{~Hz}, 1 \mathrm{H}), 7.39(\mathrm{~d}, J=7.7 \mathrm{~Hz}, 1 \mathrm{H})$, 7.45-7.58 (m, 2H), $7.58(\mathrm{~d}, J=7.7 \mathrm{~Hz}, 1 \mathrm{H}), 7.65(\mathrm{~d}, J=7.7 \mathrm{~Hz}, 1 \mathrm{H}) ;{ }^{13} \mathrm{C}$ $\operatorname{NMR}\left(75 \mathrm{MHz}, \mathrm{CDCl}_{3}\right): \delta=55.6\left(\mathrm{CH}_{3}\right), 112.5(\mathrm{CH}), 115.8(\mathrm{CH}), 119.4(\mathrm{CH})$ $119.6(\mathrm{CH}), 126.7(\mathrm{CH}), 130.0(\mathrm{CH}), 139.2(\mathrm{CH}), 139.3(\mathrm{C}), 142.3(\mathrm{C}), 158.6$ (C), 160.3 (C); IR (neat): $v=3074,2934,2835,1575,1549,1424,1217,1123$, $1037,790 \mathrm{~cm}^{-1}$; HRMS (ESI): calc. for $\mathrm{C}_{12} \mathrm{H}_{11}{ }^{79} \mathrm{BrNO}_{2}[\mathrm{M}+\mathrm{H}] \mathrm{m} / \mathrm{z} 263.9946$, found $\mathrm{m} / \mathrm{z}$ 264.0012, calc. for $\mathrm{C}_{12} \mathrm{H}_{11}{ }^{81} \mathrm{BrNO}_{2}[\mathrm{M}+\mathrm{H}] \mathrm{m} / \mathrm{z} 265.9925$, found $\mathrm{m} / \mathrm{z} 266.0001$.

2-Bromo-6-(2-fluorophenyl)pyridine (3ag): Prepared according to general procedure A by reacting 2,6-dibromopyridine ( $46 \mathrm{mg}, 0.2 \mathrm{mmol})$, with potassium 2-fluorophenyltrifluoroborate $(40 \mathrm{mg}, 0.2 \mathrm{mmol})$, sodium carbonate $(85 \mathrm{mg}, 0.8 \mathrm{mmol})$, potassium chloride $(7 \mathrm{mg}, 0.1 \mathrm{mmol})$, triphenylphosphine $\quad(6.0 \quad \mathrm{mg}, \quad 12 \quad \mathrm{~mol} \%) \quad$ and tetrakis(triphenylphosphine)palladium $(0)(7.0 \mathrm{mg}, 3 \mathrm{~mol} \%)$ in a mixture of degassed toluene/ethanol $(1.40 / 0.35 \mathrm{~mL})$ and degassed water $(0.70 \mathrm{~mL})$. The reaction was stirred at $70{ }^{\circ} \mathrm{C}$ for 4 hours. After extraction, purification of the crude material using preparative TLC $\left(20 \% \mathrm{CH}_{2} \mathrm{Cl}_{2}\right.$ in heptane) afforded $\mathbf{3 a g}$ as a white powder $(27 \mathrm{mg}, 54 \%)$. ${ }^{1} \mathrm{H}$ NMR $\left(300 \mathrm{MHz}, \mathrm{CDCl}_{3}\right): \delta=7.12$ (ddd, $J=11.6,7.9,1.2 \mathrm{~Hz}, 1 \mathrm{H}), 7.23(\mathrm{td}, J=7.9,1.2 \mathrm{~Hz}, 1 \mathrm{H}), 7.31-7.39(\mathrm{~m}, 1 \mathrm{H})$, $7.41(\mathrm{~d}, J=7.9 \mathrm{~Hz}, 1 \mathrm{H}), 7.57(\mathrm{t}, J=7.9 \mathrm{~Hz}, 1 \mathrm{H}), 7.76(\mathrm{dd}, J=7.9,1.9 \mathrm{~Hz}$, $1 \mathrm{H}), 8.01(\mathrm{td}, J=7.9,1.9 \mathrm{~Hz}, 1 \mathrm{H}) ;{ }^{13} \mathrm{C} \mathrm{NMR}\left(75 \mathrm{MHz}, \mathrm{CDCl}_{3}\right): \delta=116.4$ (d, $J=23.0 \mathrm{~Hz}, \mathrm{CH}), 123.4(\mathrm{~d}, J=10.4 \mathrm{~Hz}, \mathrm{CH}), 124.8(\mathrm{~d}, J=3.1 \mathrm{~Hz}, \mathrm{CH})$, $127.0(\mathrm{CH}), 131.3(\mathrm{~d}, J=12.1 \mathrm{~Hz}, \mathrm{CH}), 131.4(\mathrm{CH}), 138.9(\mathrm{CH}), 142.1(\mathrm{C})$, 154.3 (C), 154.5 (C), 160.7 (d, $J=251.1 \mathrm{~Hz}, \mathrm{C}$ ); IR (neat): $v=3075,2922$, 1575, 1547, 1493, 1434, 1393, 1211, 1111, 802, $790 \mathrm{~cm}^{-1}$; HRMS (ESI): calc. for $\mathrm{C}_{11} \mathrm{H}_{8}{ }^{79} \mathrm{BrFN}[\mathrm{M}+\mathrm{H}] \mathrm{m} / \mathrm{z} 251.9746$, found $\mathrm{m} / \mathrm{z} 251.9818$, calc. for $\mathrm{C}_{11} \mathrm{H}_{8}{ }^{81} \mathrm{BrFN}[\mathrm{M}+\mathrm{H}] \mathrm{m} / \mathrm{z} 253.9725$, found $\mathrm{m} / \mathrm{z} 253.9798$.

2-Bromo-6-(2-methoxyphenyl)pyridine (3ah): Prepared according to general procedure A by reacting 2,6-dibromopyridine ( $46 \mathrm{mg}, 0.2 \mathrm{mmol}$ ), with potassium 2-methoxyphenyltrifluoroborate ( $43 \mathrm{mg}, 0.2 \mathrm{mmol}$ ), sodium carbonate $(85 \mathrm{mg}, 0.8 \mathrm{mmol})$, potassium chloride $(7 \mathrm{mg}, 0.1 \mathrm{mmol})$, triphenylphosphine $\quad(3.0 \quad \mathrm{mg}, \quad 6 \quad \mathrm{~mol} \%) \quad$ and tetrakis(triphenylphosphine)palladium( 0$)(7.0 \mathrm{mg}, 3 \mathrm{~mol} \%)$ in a mixture of degassed toluene/ethanol $(1.40 / 0.35 \mathrm{~mL})$ and degassed water $(0.70 \mathrm{~mL})$. The reaction was stirred at $70{ }^{\circ} \mathrm{C}$ for 4 hours. After extraction, purification of the crude material using preparative TLC ( $30 \% \mathrm{CH}_{2} \mathrm{Cl}_{2}$ in heptane) afforded 3ah as a white powder $(37 \mathrm{mg}, 70 \%) .{ }^{1} \mathrm{H}$ NMR $\left(300 \mathrm{MHz}, \mathrm{CDCl}_{3}\right): \delta=3.85$ (s, $3 \mathrm{H}), 6.92(\mathrm{dd}, J=8.0,0.7 \mathrm{~Hz}, 1 \mathrm{H}), 7.00(\mathrm{td}, J=7.9,1.2 \mathrm{~Hz}, 1 \mathrm{H}), 7.30(\mathrm{dd}$, $J=8.0,0.7 \mathrm{~Hz}, 1 \mathrm{H}), 7.31(\mathrm{td}, J=7.9,1.9 \mathrm{~Hz}, 1 \mathrm{H}), 7.47$ (t, $J=7.9,1 \mathrm{H}), 7.78$ $(\mathrm{dd}, J=7.9,1.2 \mathrm{~Hz}, 2 \mathrm{H}) ;{ }^{13} \mathrm{C} \mathrm{NMR}\left(75 \mathrm{MHz}, \mathrm{CDCl}_{3}\right): \delta=55.8\left(\mathrm{CH}_{3}\right), 111.6$ $(\mathrm{CH}), 121.37(\mathrm{CH}), 124.1(\mathrm{CH}), 126.1(\mathrm{CH}), 127.0(\mathrm{C}), 130.8(\mathrm{CH}), 131.6$ $(\mathrm{CH}), 138.2(\mathrm{CH}), 141.6(\mathrm{C}), 146.9(\mathrm{C}), 157.2(\mathrm{C}) . \mathrm{IR}$ (neat): $v=2936,2837$, 1573, 1427, 1239, 1124, 1032, $790 \mathrm{~cm}^{-1}$; HRMS (ESI): calc. for $\mathrm{C}_{12} \mathrm{H}_{11}{ }^{79} \mathrm{BrNO}_{2}[\mathrm{M}+\mathrm{H}] \mathrm{m} / \mathrm{z}$ 263.9946, found $\mathrm{m} / \mathrm{z}$ 264.0042, calc. for $\mathrm{C}_{12} \mathrm{H}_{11}{ }^{81} \mathrm{BrNO}_{2}[\mathrm{M}+\mathrm{H}] \mathrm{m} / \mathrm{z} 265.9925$, found $\mathrm{m} / \mathrm{z} 266.0013$.

2-Bromo-6-(2-benzyloxyphenyl)pyridine (3ai): Prepared according to general procedure A by reacting 2,6-dibromopyridine ( $46 \mathrm{mg}, 0.2 \mathrm{mmol}$ ), with potassium 2-benzyloxyphenyltrifluoroborate $(58 \mathrm{mg}, 0.2 \mathrm{mmol}$ ), sodium carbonate $(85 \mathrm{mg}, 0.8 \mathrm{mmol})$, potassium chloride $(7 \mathrm{mg}, 0.1 \mathrm{mmol})$, triphenylphosphine $\quad(3.0 \quad \mathrm{mg}, \quad 12 \quad \mathrm{~mol} \%) \quad$ and tetrakis(triphenylphosphine)palladium(0) $(7.0 \mathrm{mg}, 3 \mathrm{~mol} \%)$ in a mixture of degassed toluene/ethanol $(1.40 / 0.35 \mathrm{~mL})$ and degassed water $(0.70 \mathrm{~mL})$. The reaction was stirred at $70{ }^{\circ} \mathrm{C}$ for 4 hours. After extraction, purification of the crude material using preparative TLC $\left(20 \% \mathrm{CH}_{2} \mathrm{Cl}_{2}\right.$ in heptane) afforded 3ai as a white powder $(44 \mathrm{mg}, 65 \%) .{ }^{1} \mathrm{H}$ NMR $\left(300 \mathrm{MHz}, \mathrm{CDCl}_{3}\right): \delta=5.12$ (s, $2 \mathrm{H}), 7.07(\mathrm{dd}, J=8.3,0.7 \mathrm{~Hz}, 1 \mathrm{H}), 7.12(\mathrm{td}, J=7.7,1.1 \mathrm{~Hz}, 1 \mathrm{H}), 7.25-7.40$ (m, 7H), $7.52(\mathrm{t}, J=7.7 \mathrm{~Hz}, 1 \mathrm{H}), 7.89(\mathrm{dd}, J=7.7,1.7 \mathrm{~Hz}, 1 \mathrm{H}), 7.90(\mathrm{dd}, J$ $=7.7,0.7 \mathrm{~Hz}, 1 \mathrm{H}) ;{ }^{13} \mathrm{C} \mathrm{NMR}\left(75 \mathrm{MHz}, \mathrm{CDCl}_{3}\right): \delta=70.9\left(\mathrm{CH}_{2}\right), 113.3(\mathrm{CH})$, $121.7(\mathrm{CH}), 124.2(\mathrm{CH}), 126.1(\mathrm{CH}), 126.7(\mathrm{C}), 127.3(2 \mathrm{CH}), 128.1(\mathrm{CH})$, $128.7(2 \mathrm{CH}), 130.7(\mathrm{CH}), 131.7(\mathrm{CH}), 137.0(\mathrm{C}), 138.2(\mathrm{CH}), 141.6(\mathrm{C})$, 156.4 (C), 157.2 (C); IR (neat): $v=2923,2854,1600,1573,1428,1391$, 1127, 1007, $790 \mathrm{~cm}^{-1}$; HRMS (ESI): calc. for $\mathrm{C}_{18} \mathrm{H}_{15}{ }^{79} \mathrm{BrNO}_{2}[\mathrm{M}+\mathrm{H}] \mathrm{m} / \mathrm{z}$ 340.0259 , found $\mathrm{m} / \mathrm{z} 340.0314$, calc. for $\mathrm{C}_{18} \mathrm{H}_{15}{ }^{81} \mathrm{BrNO}_{2} \quad[\mathrm{M}+\mathrm{H}] \mathrm{m} / \mathrm{z}$ 342.0296 , found $\mathrm{m} / \mathrm{z} 342.0238$.

2-Bromo-6-(3,4-dimethoxyphenyl)pyridine (3aj): Prepared according to general procedure A by reacting 2,6-dibromopyridine ( $46 \mathrm{mg}, 0.2 \mathrm{mmol}$ ), with potassium 2,4-dimethoxyphenyltrifluoroborate $(49 \mathrm{mg}, 0.2 \mathrm{mmol})$, sodium carbonate ( $85 \mathrm{mg}, 0.8 \mathrm{mmol})$, potassium chloride $(7 \mathrm{mg}, 0.1 \mathrm{mmol})$, triphenylphosphine $\quad(3.0 \quad \mathrm{mg}, \quad 6 \quad \mathrm{~mol} \quad \%) \quad$ and tetrakis(triphenylphosphine)palladium( 0$)(7.0 \mathrm{mg}, 3 \mathrm{~mol} \%)$ in a mixture of degassed toluene/ethanol $(1.40 / 0.35 \mathrm{~mL})$ and degassed water $(0.70 \mathrm{~mL})$. The reaction was stirred at $40{ }^{\circ} \mathrm{C}$ for 6 hours. After extraction, purification of the crude material using preparative TLC (20\% EtOAc in heptane) afforded 3aj as a white powder $(28 \mathrm{mg}, 47 \%) .{ }^{1} \mathrm{H}$ NMR $\left(300 \mathrm{MHz}, \mathrm{CDCl}_{3}\right): \delta=3.86(\mathrm{~s}$, $3 \mathrm{H}), 3.92(\mathrm{~s}, 3 \mathrm{H}), 6.86(\mathrm{~d}, J=8.3 \mathrm{~Hz}, 1 \mathrm{H}), 7.27(\mathrm{dd}, J=7.7,1.0 \mathrm{~Hz}, 1 \mathrm{H})$, 7.40-7.50 (m, 2H), 7.52-7.57 (m, 2H); ${ }^{13} \mathrm{C}$ NMR (75 MHz, $\left.\mathrm{CDCl}_{3}\right): \delta=56.2$ $\left(\mathrm{CH}_{3}\right), 56.3\left(\mathrm{CH}_{3}\right), 110.2(\mathrm{CH}), 111.2(\mathrm{CH}), 118.5(\mathrm{CH}), 119.9(\mathrm{CH}) 125.8$ (CH), 130.8 (C), $139.1(\mathrm{CH}), 142.2(\mathrm{C}), 149.5$ (C), 150.7 (C), 158.4 (C); IR (neat): $v=3001,2959,2934,2835,1546,1513,1420,1254,1170,1022,790$ $\mathrm{cm}^{-1}$; HRMS (ESI): calc. for $\mathrm{C}_{13} \mathrm{H}_{13}{ }^{79} \mathrm{BrNO}_{2}[\mathrm{M}+\mathrm{H}] \mathrm{m} / \mathrm{z} 294.0051$, found $\mathrm{m} / \mathrm{z}$ 294.0130, calc. for $\mathrm{C}_{13} \mathrm{H}_{13}{ }^{81} \mathrm{BrNO}_{2}[\mathrm{M}+\mathrm{H}] \mathrm{m} / \mathrm{z}$ 296.0031, found $\mathrm{m} / \mathrm{z}$ 296.0112

3-Bromo-5-(4-ethoxyphenyl)pyridine (3ba): Prepared according to general procedure A by reacting 3,5-dibromopyridine $(46 \mathrm{mg}, 0.2 \mathrm{mmol})$, with potassium 4-ethoxyphenyltrifluoroborate ( $46 \mathrm{mg}, 0.2 \mathrm{mmol})$, sodium carbonate $(85 \mathrm{mg}, 0.8 \mathrm{mmol})$, potassium chloride $(7 \mathrm{mg}, 0.1 \mathrm{mmol})$, triphenylphosphine $\quad(3.0 \quad \mathrm{mg}, \quad 6 \quad \mathrm{~mol} \%) \quad$ and tetrakis(triphenylphosphine)palladium(0) $(7.0 \mathrm{mg}, 3 \mathrm{~mol} \%)$ in a mixture of degassed toluene/ethanol $(1.40 / 0.35 \mathrm{~mL})$ and degassed water $(0.70 \mathrm{~mL})$. The reaction was stirred at $40{ }^{\circ} \mathrm{C}$ for 6 hours. After extraction, purification of the crude material by flash chromatography on silica gel ( $0 \%$ to $20 \%$ EtOAc in heptane) afforded $3 \mathbf{b a}$ as a white powder $(42 \mathrm{mg}, 76 \%) .{ }^{1} \mathrm{H} \mathrm{NMR}(300 \mathrm{MHz}$, $\left.\mathrm{CDCl}_{3}\right): \delta=1.38(\mathrm{t}, J=7.0 \mathrm{~Hz}, 3 \mathrm{H}), 4.02(\mathrm{q}, J=7.0 \mathrm{~Hz}, 2 \mathrm{H}), 6.92(\mathrm{~d}, J=$ $8.8 \mathrm{~Hz}, 2 \mathrm{H}), 7.41(\mathrm{~d}, J=8.8 \mathrm{~Hz}, 2 \mathrm{H}), 7.90-7.96(\mathrm{~m}, 1 \mathrm{H}), 8.23-9.07(\mathrm{~m}, 2 \mathrm{H})$; ${ }^{13} \mathrm{C} \mathrm{NMR}(75 \mathrm{MHz}, \mathrm{CDCl} 3): \delta=15.0\left(\mathrm{CH}_{3}\right), 63.9\left(\mathrm{CH}_{2}\right), 115.4(3 \mathrm{CH}), 128.5$ (3CH), 128.7 (C), $136.6(\mathrm{CH}), 146.1$ (C), 148.7 (C), 159.8 (C); IR (neat): $v$ = 2975, 2926, 1606, 1575, 1547, 1512, 1433, 1421, 1392, 1251, 1115, 1045, $790 \mathrm{~cm}^{-1}$; HRMS (ESI): calc. for $\mathrm{C}_{13} \mathrm{H}_{13}{ }^{79} \mathrm{BrNO}[\mathrm{M}+\mathrm{H}] \mathrm{m} / \mathrm{z} 278.0102$, found $\mathrm{m} / \mathrm{z}$ 278.0120, calc. for $\mathrm{C}_{13} \mathrm{H}_{13}{ }^{81} \mathrm{BrNO}[\mathrm{M}+\mathrm{H}] \mathrm{m} / \mathrm{z} 280.0082$, found $\mathrm{m} / \mathrm{z}$ 280.0113 .

3-Bromo-5-(4-methylphenyl)pyridine (3bb): Prepared according to general procedure A by reacting 3,5-dibromopyridine ( $46 \mathrm{mg}, 0.2 \mathrm{mmol}$ ), 
with potassium 4-methylphenyltrifluoroborate $(40 \mathrm{mg}, 0.2 \mathrm{mmol}$ ), sodium carbonate $(85 \mathrm{mg}, 0.8 \mathrm{mmol})$, potassium chloride $(7 \mathrm{mg}, 0.1 \mathrm{mmol})$, triphenylphosphine $\quad(3.0 \quad \mathrm{mg}, \quad 6 \quad \mathrm{~mol} \%) \quad$ and tetrakis(triphenylphosphine)palladium( $(0)(7.0 \mathrm{mg}, 3 \mathrm{~mol} \%)$ in a mixture of degassed toluene/ethanol $(1.40 / 0.35 \mathrm{~mL})$ and degassed water $(0.70 \mathrm{~mL})$. The reaction was stirred at $40{ }^{\circ} \mathrm{C}$ for 6 hours. After extraction, purification of the crude material by flash chromatography on silica gel (0\% to 20\% EtOAc in heptane) afforded $\mathbf{3 b b}$ as a white powder $(37 \mathrm{mg}, 76 \%) .{ }^{1} \mathrm{H} \mathrm{NMR}(300 \mathrm{MHz}$, $\left.\mathrm{CDCl}_{3}\right): \delta=2.39$ (s, 3H), $7.27(\mathrm{~d}, J=8.1 \mathrm{~Hz}, 2 \mathrm{H}), 7.43(\mathrm{~d}, J=8.1 \mathrm{~Hz}, 2 \mathrm{H})$, 7.97-7.98 (m, 1H), $8.61(\mathrm{~s}, 1 \mathrm{H}), 8.72(\mathrm{~s}, 1 \mathrm{H}) ;{ }^{13} \mathrm{C} \mathrm{NMR}\left(75 \mathrm{MHz}, \mathrm{CDCl}_{3}\right): \delta$ $=21.4\left(\mathrm{CH}_{3}\right), 121.1(\mathrm{C}), 127.2(2 \mathrm{CH}), 130.1(2 \mathrm{CH}), 133.6(\mathrm{C}), 136.8(\mathrm{CH})$, $138.4(\mathrm{C}), 139.0(\mathrm{C}), 146.4(\mathrm{CH}), 149.2(\mathrm{CH})$; IR (neat): $v=3024,2917$, 2854, 1739, 1433, 1377, 1218, 1107, 1008, $825 \mathrm{~cm}^{-1}$; HRMS (ESI): calc. for $\mathrm{C}_{12} \mathrm{H}_{11}{ }^{79} \mathrm{BrN}[\mathrm{M}+\mathrm{H}] \mathrm{m} / \mathrm{z} 247.9997$, found $\mathrm{m} / \mathrm{z} 248.0088$, calc. for $\mathrm{C}_{12} \mathrm{H}_{11}{ }^{81} \mathrm{BrN}[\mathrm{M}+\mathrm{H}] \mathrm{m} / \mathrm{z} 249.9976$, found $\mathrm{m} / \mathrm{z} 250.0066$.

3-Bromo-5-(4-nitrophenyl)pyridine (3bc): Prepared according to general procedure A by reacting 3,5-dibromopyridine $(46 \mathrm{mg}, 0.2 \mathrm{mmol})$, with potassium 4-nitrophenyltrifluoroborate $(46 \mathrm{mg}, 0.2 \mathrm{mmol})$, sodium carbonate $(85 \mathrm{mg}, 0.8 \mathrm{mmol})$, potassium chloride $(7 \mathrm{mg}, 0.1 \mathrm{mmol})$, triphenylphosphine $\quad(3.0 \quad \mathrm{mg}, \quad 6 \quad \mathrm{~mol} \%) \quad$ and tetrakis(triphenylphosphine)palladium( $(0)(7.0 \mathrm{mg}, 3 \mathrm{~mol} \%)$ in a mixture of degassed toluene/ethanol $(1.40 / 0.35 \mathrm{~mL})$ and degassed water $(0.70 \mathrm{~mL})$. The reaction was stirred at $40{ }^{\circ} \mathrm{C}$ for 6 hours. After extraction, purification of the crude material by flash chromatography on silica gel ( $0 \%$ to $20 \%$ EtOAc in heptane) afforded $3 \mathbf{b c}$ as a yellow powder (31 mg, 64\%). ${ }^{1} \mathrm{H}$ NMR $(300 \mathrm{MHz}$ $\left.\mathrm{CDCl}_{3}\right): \delta=7.73(\mathrm{~d}, J=8.8 \mathrm{~Hz}, 2 \mathrm{H}), 8.10(\mathrm{t}, J=2.0 \mathrm{~Hz}, 1 \mathrm{H}), 8.34(\mathrm{~d}, J=$ $8.8 \mathrm{~Hz}, 2 \mathrm{H}), 8.77(\mathrm{dd}, J=15.7,2.0 \mathrm{~Hz}, 2 \mathrm{H}) ;{ }^{13} \mathrm{C} \mathrm{NMR}\left(75 \mathrm{MHz}, \mathrm{CDCl}_{3}\right): \delta$ $=121.6(\mathrm{C}), 124.8(2 \mathrm{CH}), 128.4(2 \mathrm{CH}), 136.4(\mathrm{C}), 138.0(\mathrm{CH}), 142.5(\mathrm{C})$, $145.9(\mathrm{CH}), 148.4(\mathrm{C}), 150.4(\mathrm{CH})$; IR (neat): $v=3109,3085,2963,1511$, 1343, 1259, 1089, 1047, 1015, $790 \mathrm{~cm}^{-1}$; HRMS (ESI): calc. for $\mathrm{C}_{11} \mathrm{H}_{8}{ }^{79} \mathrm{BrN}_{2} \mathrm{O}_{2} \quad[\mathrm{M}+\mathrm{H}] \mathrm{m} / \mathrm{z}$ 278.9691, found $\mathrm{m} / \mathrm{z}$ 278.9761, calc. for $\mathrm{C}_{11} \mathrm{H}_{8}{ }^{81} \mathrm{BrN}_{2} \mathrm{O}_{2}[\mathrm{M}+\mathrm{H}] \mathrm{m} / \mathrm{z} 280.9670$, found $\mathrm{m} / \mathrm{z} 280.9768$.

1-Bromo-3-(4-ethoxyphenyl)benzene (3ca): Prepared according to general procedure A by reacting 1,3-dibromobenzene $(24 \mu \mathrm{L}, 0.2 \mathrm{mmol})$, with potassium 4-ethoxyphenyltrifluoroborate ( $46 \mathrm{mg}, 0.2 \mathrm{mmol}$ ), sodium carbonate $(85 \mathrm{mg}, 0.8 \mathrm{mmol})$, potassium chloride $(7 \mathrm{mg}, 0.1 \mathrm{mmol})$, triphenylphosphine $\quad(3.0 \quad \mathrm{mg}, \quad 6.0 \quad \mathrm{~mol} \quad \%) \quad$ and tetrakis(triphenylphosphine)palladium $(0)(7.0 \mathrm{mg}, 3 \mathrm{~mol} \%)$ in a mixture of degassed toluene/ethanol $(1.40 / 0.35 \mathrm{~mL})$ and degassed water $(0.70 \mathrm{~mL})$. The reaction was stirred at $40{ }^{\circ} \mathrm{C}$ for 6 hours. After extraction, purification of the crude material by preparative TLC $\left(20 \% \mathrm{CH}_{2} \mathrm{Cl}_{2}\right.$ in heptane) afforded 3ca as a white powder $(45 \mathrm{mg}, 82 \%) ;{ }^{1} \mathrm{H} \mathrm{NMR}\left(300 \mathrm{MHz}, \mathrm{CDCl}_{3}\right): \delta=1.36(\mathrm{t}, J=$ $7.0 \mathrm{~Hz}, 3 \mathrm{H}), 3.99(\mathrm{q}, J=7.0 \mathrm{~Hz}, 2 \mathrm{H}), 6.88(\mathrm{~d}, J=8.8 \mathrm{~Hz}, 2 \mathrm{H}), 7.15-7.22(\mathrm{~m}$, $1 \mathrm{H}), 7.30-7.38(\mathrm{~m}, 2 \mathrm{H}), 7.40(\mathrm{~d}, J=8.8 \mathrm{~Hz}, 2 \mathrm{H}), 7.61(\mathrm{t}, J=1.9 \mathrm{~Hz}, 1 \mathrm{H})$; ${ }^{13} \mathrm{C}$ NMR $\left(75 \mathrm{MHz}, \mathrm{CDCl}_{3}\right): \delta=15.1\left(\mathrm{CH}_{3}\right), 63.7\left(\mathrm{CH}_{2}\right), 115.0(2 \mathrm{CH}), 123.1$ (C), $125.5(\mathrm{CH}), 128.3(2 \mathrm{CH}), 129.7(\mathrm{CH}), 129.9(\mathrm{CH}), 130.4(\mathrm{CH}), 132.2$ (C), 143.2 (C), 159.1 (C); IR (neat): $v=2977,2929,1606,1579,1469,1393$, 1251, 1188, 1047, $790 \mathrm{~cm}^{-1}$; HRMS (APPI): calc. for $\mathrm{C}_{14} \mathrm{H}_{13}{ }^{79} \mathrm{BrO}\left[\mathrm{M}^{+}\right] \mathrm{m} / \mathrm{z}$ 276.0150, found $\mathrm{m} / \mathrm{z} 276.0166$, calc. for $\mathrm{C}_{14} \mathrm{H}_{13}{ }^{81} \mathrm{BrO}\left[\mathrm{M}^{+}\right] \mathrm{m} / \mathrm{z} 278.0129$, found $\mathrm{m} / \mathrm{z} 278.0147$.

1-Bromo-3-(4-methylphenyl)benzene (3cb): Prepared according to general procedure A by reacting 1,3-dibromobenzene $(24 \mu \mathrm{L}, 0.2 \mathrm{mmol})$, with potassium 4-methylphenyltrifluoroborate ( $40 \mathrm{mg}, 0.2 \mathrm{mmol}$ ), sodium carbonate $(85 \mathrm{mg}, 0.8 \mathrm{mmol})$, potassium chloride $(7 \mathrm{mg}, 0.1 \mathrm{mmol})$, triphenylphosphine $\quad(3.0 \quad \mathrm{mg}, \quad 6 \quad \mathrm{~mol} \quad \%) \quad$ and tetrakis(triphenylphosphine)palladium( 0$)(7.0 \mathrm{mg}, 3 \mathrm{~mol} \%)$ in a mixture of degassed toluene/ethanol $(1.40 / 0.35 \mathrm{~mL})$ and degassed water $(0.70 \mathrm{~mL})$. The reaction was stirred at $40{ }^{\circ} \mathrm{C}$ for 6 hours. After extraction, purification of the crude material by preparative TLC $\left(20 \% \mathrm{CH}_{2} \mathrm{Cl}_{2}\right.$ in heptane) afforded $3 \mathbf{c b}$ as a beige powder (38 mg, $77 \%)$. ${ }^{1} \mathrm{H} \mathrm{NMR}\left(300 \mathrm{MHz}, \mathrm{CDCl}_{3}\right): \delta=2.37(\mathrm{~s}, 3 \mathrm{H})$, 7.19-7.25 (m, 2H), 7.27 (d, $J=7.9 \mathrm{~Hz}, 1 \mathrm{H}), 7.39-7.44(\mathrm{~m}, 3 \mathrm{H}), 7.45-7.49$ (m, $1 \mathrm{H}), 7.69(\mathrm{t}, J=1.9 \mathrm{~Hz}, 1 \mathrm{H}) ;{ }^{13} \mathrm{C} \mathrm{NMR}\left(75 \mathrm{MHz}, \mathrm{CDCl}_{3}\right): \delta=21.3\left(\mathrm{CH}_{3}\right)$, $123.1(\mathrm{C}), 125.7(\mathrm{CH}), 127.1(2 \mathrm{CH}), 129.8(2 \mathrm{CH}), 130.1(\mathrm{CH}), 130.2(\mathrm{CH})$,
$130.4(\mathrm{CH}), 137.0(\mathrm{C}), 138.0(\mathrm{C}), 143.5(\mathrm{C})$; IR (neat): $v=3032,2857,1555$, 1467, 1450, 1030, 822, $790 \mathrm{~cm}^{-1}$; HRMS (APPI): calc. for $\mathrm{C}_{13} \mathrm{H}_{11}{ }^{79} \mathrm{Br}\left[\mathrm{M}^{+}\right]$ $\mathrm{m} / \mathrm{z} 246.0044$, found $\mathrm{m} / \mathrm{z} 246.0032$, calc. for $\mathrm{C}_{13} \mathrm{H}_{11}{ }^{81} \mathrm{Br}\left[\mathrm{M}^{+}\right] \mathrm{m} / \mathrm{z} 248.0024$, found $\mathrm{m} / \mathrm{z} 248.0013$.

1-Bromo-3-(4-nitrophenyl)benzene (3cc): Prepared according to general procedure A by reacting 1,3-dibromobenzene ( $24 \mu \mathrm{L}, 0.2 \mathrm{mmol})$, with potassium 4-nitrophenyltrifluoroborate $(46 \mathrm{mg}, 0.2 \mathrm{mmol})$, sodium carbonate $(85 \mathrm{mg}, 0.8 \mathrm{mmol})$, potassium chloride $(7 \mathrm{mg}, 0.1 \mathrm{mmol}$ ), triphenylphosphine (3.0 $\mathrm{mg}, \quad 6 \quad \mathrm{~mol} \%) \quad$ and tetrakis(triphenylphosphine)palladium( $(0)(7.0 \mathrm{mg}, 3 \mathrm{~mol} \%)$ in a mixture of degassed toluene/ethanol $(1.40 / 0.35 \mathrm{~mL})$ and degassed water $(0.70 \mathrm{~mL})$. The reaction was stirred at $40{ }^{\circ} \mathrm{C}$ for 6 hours. After extraction, purification of the crude material by preparative TLC $\left(20 \% \mathrm{CH}_{2} \mathrm{Cl}_{2}\right.$ in heptane) afforded $3 \mathbf{c c}$ as a beige powder $(26 \mathrm{mg}, 47 \%) ;{ }^{1} \mathrm{H} \mathrm{NMR}\left(300 \mathrm{MHz}, \mathrm{CDCl}_{3}\right): \delta=7.35$ (dd, $J$ $=7.9,7.6 \mathrm{~Hz}, 1 \mathrm{H}), 7.49-7.59(\mathrm{~m}, 2 \mathrm{H}), 7.69(\mathrm{~d}, J=8.8 \mathrm{~Hz}, 2 \mathrm{H}), 7.75(\mathrm{t}, J=$ $1.8 \mathrm{~Hz}, 1 \mathrm{H}), 8.29(\mathrm{~d}, J=8.8 \mathrm{~Hz}, 2 \mathrm{H}) ;{ }^{13} \mathrm{C} \mathrm{NMR}\left(75 \mathrm{MHz}, \mathrm{CDCl}_{3}\right): \delta=123.5$ (C), $124.4(2 \mathrm{CH}), 126.2(\mathrm{CH}), 128.1(2 \mathrm{CH}), 130.7(\mathrm{CH}), 130.9(\mathrm{CH}), 131.6$ (C), $132.1(\mathrm{CH}), 141.1(\mathrm{C}), 146.2(\mathrm{C})$; IR (neat): $v=2988,2901,1511,1406$, 1394, 1343, 1242, 1074, 1056, $854 \mathrm{~cm}^{-1}$; MS: no ionization could be observed for this compound.

1-Bromo-4-(4-ethoxyphenyl)benzene (3da): Prepared according to general procedure A by reacting 1,4-dibromobenzene ( $47 \mathrm{mg}, 0.2 \mathrm{mmol}$ ), with potassium 4-ethoxyphenyltrifluoroborate $(46 \mathrm{mg}, 0.2 \mathrm{mmol})$, sodium carbonate $(85 \mathrm{mg}, 0.8 \mathrm{mmol})$, potassium chloride $(7 \mathrm{mg}, 0.1 \mathrm{mmol})$, triphenylphosphine $\quad(3.0 \quad \mathrm{mg}, \quad 6 \quad \mathrm{~mol} \%) \quad$ and tetrakis(triphenylphosphine)palladium( $(0)(7.0 \mathrm{mg}, 3 \mathrm{~mol} \%)$ in a mixture of degassed toluene/ethanol $(1.40 / 0.35 \mathrm{~mL})$ and degassed water $(0.70 \mathrm{~mL})$. The reaction was stirred at $40{ }^{\circ} \mathrm{C}$ for 6 hours. After extraction, purification of the crude material by preparative TLC ( $20 \% \mathrm{CH}_{2} \mathrm{Cl}_{2}$ in heptane) afforded $\mathbf{3 d a}$ as a white powder (42 mg, $76 \%$ ). ${ }^{1} \mathrm{H}$ NMR (300 MHz, $\left.\mathrm{CDCl}_{3}\right): \delta=1.43$ (t, $J=$ $7.0 \mathrm{~Hz}, 3 \mathrm{H}), 4.06$ (q, $J=7.0 \mathrm{~Hz}, 2 \mathrm{H}), 6.95(\mathrm{~d}, J=8.6 \mathrm{~Hz}, 2 \mathrm{H}), 7.39$ (d, $J=$ $8.6 \mathrm{~Hz}, 2 \mathrm{H}), 7.46(\mathrm{~d}, J=8.6 \mathrm{~Hz}, 2 \mathrm{H}), 7.51(\mathrm{~d}, J=8.6 \mathrm{~Hz}, 2 \mathrm{H}) ;{ }^{13} \mathrm{C} \mathrm{NMR}(75$ $\left.\mathrm{MHz}, \mathrm{CDCl}_{3}\right): \delta=14.9\left(\mathrm{CH}_{3}\right), 63.6\left(\mathrm{CH}_{2}\right), 114.9(2 \mathrm{CH}), 120.7(\mathrm{C}), 127.7$ (C), $128.0(2 \mathrm{CH}), 128.3(2 \mathrm{CH}), 131.8(2 \mathrm{CH}), 139.8(\mathrm{C}), 158.8(\mathrm{C})$; IR (neat): $v=2980,2929,1738,1604,1475,1390,1283,1248,1197,1045,815,800$ $\mathrm{cm}^{-1}$; HRMS (APPI): calc. for $\mathrm{C}_{14} \mathrm{H}_{13}{ }^{79} \mathrm{BrO}\left[\mathrm{M}^{+}\right] \mathrm{m} / \mathrm{z} 276.0150$, found $\mathrm{m} / \mathrm{z}$ 276.0134, calc. for $\mathrm{C}_{14} \mathrm{H}_{13}{ }^{81} \mathrm{BrO}\left[\mathrm{M}^{+}\right] \mathrm{m} / \mathrm{z}$ 278.0129, found $\mathrm{m} / \mathrm{z} 278.0115$.

1-Bromo-4-(4-methylphenyl)benzene (3db): Prepared according to general procedure A by reacting 1,4-dibromobenzene ( $47 \mathrm{mg}, 0.2 \mathrm{mmol}$ ), with potassium 4-methylphenyltrifluoroborate ( $40 \mathrm{mg}, 0.2 \mathrm{mmol}$ ), sodium carbonate $(85 \mathrm{mg}, 0.8 \mathrm{mmol})$, potassium chloride $(7 \mathrm{mg}, 0.1 \mathrm{mmol}$ ), triphenylphosphine $\quad(3.0 \quad \mathrm{mg}, \quad 6 \quad \mathrm{~mol} \%) \quad$ and tetrakis(triphenylphosphine)palladium( $(0)(7.0 \mathrm{mg}, 3 \mathrm{~mol} \%)$ in a mixture of degassed toluene/ethanol $(1.40 / 0.35 \mathrm{~mL})$ and degassed water $(0.70 \mathrm{~mL})$. The reaction was stirred at $40{ }^{\circ} \mathrm{C}$ for 6 hours. After extraction, purification of the crude material by preparative TLC ( $20 \% \mathrm{CH}_{2} \mathrm{Cl}_{2}$ in heptane) afforded $\mathbf{3 d b}$ as a white powder $(36 \mathrm{mg}, 73 \%) .{ }^{1} \mathrm{H} \mathrm{NMR}\left(300 \mathrm{MHz}, \mathrm{CDCl}_{3}\right): \delta=2.37(\mathrm{~s}, 3 \mathrm{H})$, $7.23(\mathrm{~d}, J=8.2 \mathrm{~Hz}, 2 \mathrm{H}), 7.42(\mathrm{~d}, J=8.6 \mathrm{~Hz}, 2 \mathrm{H}), 7.43(\mathrm{~d}, J=8.2 \mathrm{~Hz}, 2 \mathrm{H})$, $7.52(\mathrm{~d}, J=8.6 \mathrm{~Hz}, 2 \mathrm{H}) ;{ }^{13} \mathrm{C} \mathrm{NMR}\left(75 \mathrm{MHz}, \mathrm{CDCl}_{3}\right): \delta=21.3\left(\mathrm{CH}_{3}\right), 121.4$ (C), $127.0(2 \mathrm{CH}), 128.8(2 \mathrm{CH}), 129.7(\mathrm{C}), 140.3(\mathrm{C}), 129.8(2 \mathrm{CH}), 132.0$ (2CH), 137.7 (C); IR (neat): $v=3030,2855,1555,1462,1450,1025,821$, $790 \mathrm{~cm}^{-1}$; HRMS (APPI): calc. for $\mathrm{C}_{13} \mathrm{H}_{11}{ }^{79} \mathrm{Br}\left[\mathrm{M}^{+}\right] \mathrm{m} / \mathrm{z} 246.0044$, found $\mathrm{m} / \mathrm{z}$ 246.0064, calc. for $\mathrm{C}_{13} \mathrm{H}_{11}{ }^{81} \mathrm{Br}\left[\mathrm{M}^{+}\right] \mathrm{m} / \mathrm{z} 248.0024$, found $\mathrm{m} / \mathrm{z} 248.0044$.

1-Bromo-4-(4-nitrophenyl)benzene (3dc): Prepared according to general procedure A by reacting 1,4-dibromobenzene ( $47 \mathrm{mg}, 0.2 \mathrm{mmol})$, with potassium 4-nitrophenyltrifluoroborate $(46 \mathrm{mg}, 0.2 \mathrm{mmol})$, sodium carbonate $(85 \mathrm{mg}, 0.8 \mathrm{mmol})$, potassium chloride $(7 \mathrm{mg}, 0.1 \mathrm{mmol})$, triphenylphosphine $\quad(3.0 \quad \mathrm{mg}, \quad 6 \quad \mathrm{~mol} \quad \%) \quad$ and tetrakis(triphenylphosphine)palladium( $(0)(7.0 \mathrm{mg}, 3 \mathrm{~mol} \%)$ in a mixture of degassed toluene/ethanol $(1.40 / 0.35 \mathrm{~mL})$ and degassed water $(0.70 \mathrm{~mL})$. The reaction was stirred at $40{ }^{\circ} \mathrm{C}$ for 6 hours. After extraction, purification of the crude material by preparative TLC $\left(20 \% \mathrm{CH}_{2} \mathrm{Cl}_{2}\right.$ in heptane) afforded $\mathbf{3 d c}$ as a beige powder (30 mg, $55 \%) .{ }^{1} \mathrm{H} \mathrm{NMR}\left(300 \mathrm{MHz}, \mathrm{CDCl}_{3}\right): \delta=7.47$ (d, $J=$ 
$8.8 \mathrm{~Hz}, 2 \mathrm{H}), 7.61(\mathrm{~d}, J=8.8 \mathrm{~Hz}, 2 \mathrm{H}), 7.69(\mathrm{~d}, J=8.8 \mathrm{~Hz}, 2 \mathrm{H}), 8.28(\mathrm{~d}, J=$ $8.8 \mathrm{~Hz}, 2 \mathrm{H}) ;{ }^{13} \mathrm{C} \mathrm{NMR}\left(75 \mathrm{MHz}, \mathrm{CDCl}_{3}\right): \delta=123.7(\mathrm{C}), 124.5(2 \mathrm{CH}), 127.9$ $(2 \mathrm{CH}), 129.1(2 \mathrm{CH}), 132.6(2 \mathrm{CH}), 137.9(\mathrm{C}), 146.6(\mathrm{C}), 147.5(\mathrm{C})$; IR (neat): $v=2998,2951,1509,1406,1394,1346,1245,1074,1056,871 \mathrm{~cm}^{-1}$; MS: no ionization could be observed for this compound.

1-Bromo-2-(4-ethoxyphenyl)benzene (3ea): Prepared according to general procedure A by reacting 1,2-dibromobenzene $(24 \mu \mathrm{L}, 0.2 \mathrm{mmol})$, with potassium 4-ethoxyphenyltrifluoroborate ( $46 \mathrm{mg}, 0.2 \mathrm{mmol}$ ), sodium carbonate $(85 \mathrm{mg}, 0.8 \mathrm{mmol})$, potassium chloride $(7 \mathrm{mg}, 0.1 \mathrm{mmol})$, triphenylphosphine $\quad(3.0 \quad \mathrm{mg}, \quad 6 \quad \mathrm{~mol} \%) \quad$ and tetrakis(triphenylphosphine)palladium $(0)(7.0 \mathrm{mg}, 3 \mathrm{~mol} \%)$ in a mixture of degassed toluene/ethanol $(1.40 / 0.35 \mathrm{~mL})$ and degassed water $(0.70 \mathrm{~mL})$. The reaction was stirred at $100{ }^{\circ} \mathrm{C}$ for 6 hours. The reaction was stirred at $100{ }^{\circ} \mathrm{C}$ for 6 hours. After extraction, purification of the crude material by preparative TLC (10\% EtOAc in heptane) afforded 3ea as a white powder (41 mg, 74\%). ${ }^{1} \mathrm{H}$ NMR $\left(300 \mathrm{MHz}, \mathrm{CDCl}_{3}\right): \delta=1.43(\mathrm{t}, J=7.0 \mathrm{~Hz}, 3 \mathrm{H}), 4.07(\mathrm{q}, J=7.0 \mathrm{~Hz}$, $2 \mathrm{H}), 6.94(\mathrm{~d}, J=8.7 \mathrm{~Hz}, 2 \mathrm{H}), 7.10-7.20(\mathrm{~m}, 1 \mathrm{H}), 7.27-7.31(\mathrm{~m}, 2 \mathrm{H}), 7.32(\mathrm{~d}$, $J=8.7 \mathrm{~Hz}, 2 \mathrm{H}), 7.64(\mathrm{~d}, J=8.2 \mathrm{~Hz}, 1 \mathrm{H}) ;{ }^{13} \mathrm{C} \mathrm{NMR}\left(75 \mathrm{MHz}, \mathrm{CDCl}_{3}\right): \delta=$ $15.1\left(\mathrm{CH}_{3}\right), 63.7\left(\mathrm{CH}_{2}\right), 114.1(2 \mathrm{CH}), 123.1(\mathrm{C}), 127.5(\mathrm{CH}), 128.6(\mathrm{CH})$, $130.7(2 \mathrm{CH}), 131.6(\mathrm{CH}), 133.3(\mathrm{CH}), 133.6(\mathrm{C}), 142.5(\mathrm{C}), 158.7(\mathrm{C})$; IR (neat): $v=2971,2925,1601,1570,1469,1393,1251,1181,1044,790 \mathrm{~cm}^{-1}$; HRMS (APPI): calc. for $\mathrm{C}_{14} \mathrm{H}_{13}{ }^{78} \mathrm{BrO}[\mathrm{M}+] \mathrm{m} / \mathrm{z} 276.0150$, found $\mathrm{m} / \mathrm{z}$ 276.0135, calc. for $\mathrm{C}_{14} \mathrm{H}_{13}{ }^{81} \mathrm{BrO}[\mathrm{M}+] \mathrm{m} / \mathrm{z} 278.0129$, found $\mathrm{m} / \mathrm{z} 278.0115$.

1-Bromo-2-(4-methylphenyl)benzene (3eb): Prepared according to general procedure A by reacting 1,2-dibromobenzene $(24 \mu \mathrm{L}, 0.2 \mathrm{mmol})$, with potassium 4-methylphenyltrifluoroborate ( $40 \mathrm{mg}, 0.2 \mathrm{mmol}$ ), sodium carbonate $(85 \mathrm{mg}, 0.8 \mathrm{mmol})$, potassium chloride $(7 \mathrm{mg}, 0.1 \mathrm{mmol})$, triphenylphosphine $\quad(3.0 \quad \mathrm{mg}, \quad 6 \quad \mathrm{~mol} \%) \quad$ and tetrakis(triphenylphosphine)palladium( $(0)(7.0 \mathrm{mg}, 3 \mathrm{~mol} \%)$ in a mixture of degassed toluene/ethanol $(1.40 / 0.35 \mathrm{~mL})$ and degassed water $(0.70 \mathrm{~mL})$. The reaction was stirred at $100{ }^{\circ} \mathrm{C}$ for 6 hours. After extraction, purification of the crude material by preparative TLC $\left(4 \% \mathrm{CH}_{2} \mathrm{Cl}_{2}\right.$ in heptane) afforded $3 \mathbf{e b}$ as a white powder $(20 \mathrm{mg}, 40 \%) .{ }^{1} \mathrm{H}$ NMR $\left(300 \mathrm{MHz}, \mathrm{CDCl}_{3}\right): \delta=2.44$ (s, $3 \mathrm{H})$, 7.17-7.24 (m, 1H), 7.27-7.30 (m, 1H), 7.31-7.41 (m, 4H), $7.69(\mathrm{~d}, J=$ $8.0 \mathrm{~Hz}, 2 \mathrm{H}) ;{ }^{13} \mathrm{C}$ NMR $\left(75 \mathrm{MHz}, \mathrm{CDCl}_{3}\right): \delta=21.5\left(\mathrm{CH}_{3}\right), 122.9(\mathrm{C}), 127.5$ $(\mathrm{CH}), 128.7(\mathrm{CH}), 128.9(2 \mathrm{CH}), 129.5(2 \mathrm{CH}), 131.5(\mathrm{CH}), 133.3(\mathrm{CH}), 137.6$ (C), 138.4 (C), 142.8 (C); IR (neat): $v=3029,2847,1551,1459,1450,1025$, 817, $790 \mathrm{~cm}^{-1}$; HRMS (APPI): calc. for $\mathrm{C}_{13} \mathrm{H}_{11}{ }^{78} \mathrm{Br}[\mathrm{M}+] \mathrm{m} / \mathrm{z} 246.0044$, found $\mathrm{m} / \mathrm{z} 246.0036$, calc. for $\mathrm{C}_{13} \mathrm{H}_{11}{ }^{81} \mathrm{Br}[\mathrm{M}+] \mathrm{m} / \mathrm{z} 248.0024$, found $\mathrm{m} / \mathrm{z}$ 248.0017 .

1-Bromo-2-(4-nitrophenyl)benzene (3ec): Prepared according to general procedure A by reacting 1,2-dibromobenzene ( $24 \mu \mathrm{L}, 0.2 \mathrm{mmol})$, with potassium 4-nitrophenyltrifluoroborate $(46 \mathrm{mg}, 0.2 \mathrm{mmol})$, sodium carbonate $(85 \mathrm{mg}, 0.8 \mathrm{mmol})$, potassium chloride $(7 \mathrm{mg}, 0.1 \mathrm{mmol})$, triphenylphosphine $\quad(3.0 \quad \mathrm{mg}, \quad 6 \quad \mathrm{~mol} \%) \quad$ and tetrakis(triphenylphosphine)palladium( $(0)(7.0 \mathrm{mg}, 3 \mathrm{~mol} \%)$ in a mixture of degassed toluene/ethanol $(1.40 / 0.35 \mathrm{~mL})$ and degassed water $(0.70 \mathrm{~mL})$. The reaction was stirred at $100{ }^{\circ} \mathrm{C}$ for 6 hours. After extraction, purification of the crude material by preparative TLC (10\% EtOAc in heptane) in heptane afforded 3ec as a white powder (27 mg, $49 \%)$. ${ }^{1} \mathrm{H} \mathrm{NMR} \mathrm{(300} \mathrm{MHz,} \mathrm{CDCl}_{3}$ ): $\delta=7.25-7.34(\mathrm{~m}, 2 \mathrm{H}), 7.40(\mathrm{ddd}, J=8.0,6.8,1.1 \mathrm{~Hz}, 1 \mathrm{H}), 7.57(\mathrm{~d}, J=8.8$ $\mathrm{Hz}, 2 \mathrm{H}), 7.69\left(\mathrm{dd},(\mathrm{d}, J=8.0,1.1 \mathrm{~Hz}, 1 \mathrm{H}), 8.28(\mathrm{~d}, J=8.8 \mathrm{~Hz}, 2 \mathrm{H}) ;{ }^{13} \mathrm{C}\right.$ NMR $\left(75 \mathrm{MHz}, \mathrm{CDCl}_{3}\right): \delta=122.0(\mathrm{C}), 123.3(2 \mathrm{CH}), 127.7(\mathrm{CH}), 129.9(\mathrm{CH})$, $130.5(2 \mathrm{CH}), 130.9(\mathrm{CH}), 133.5(\mathrm{CH}), 140.4(\mathrm{C}), 147.5(\mathrm{C}), 153.5(\mathrm{C})$; IR (neat): $v=2985,2888,1509,1401,1394,1340,1242,1074,1045,844 \mathrm{~cm}^{-1}$; MS: no ionization could be observed for this compound.

2-Bromo-5-(4-ethoxyphenyl)thiophene (3fa): Prepared according to general procedure A by reacting 2,5-dibromothiophene ( $23 \mu \mathrm{L}, 0.2 \mathrm{mmol})$, with potassium 4-ethoxyphenyltrifluoroborate $(69 \mathrm{mg}, 0.3 \mathrm{mmol})$, sodium carbonate $(85 \mathrm{mg}, 0.8 \mathrm{mmol})$, potassium chloride $(7 \mathrm{mg}, 0.1 \mathrm{mmol})$, triphenylphosphine $\quad(3.0 \quad \mathrm{mg}, \quad 6 \quad \mathrm{~mol} \quad \%) \quad$ and tetrakis(triphenylphosphine)palladium $(0)(7.0 \mathrm{mg}, 3 \mathrm{~mol} \%)$ in a mixture of degassed toluene/ethanol $(1.40 / 0.35 \mathrm{~mL})$ and degassed water $(0.70 \mathrm{~mL})$. The reaction was stirred at $70{ }^{\circ} \mathrm{C}$ overnight. After extraction, purification of the crude material by flash chromatography on neutral alumina gel (2\% MTBE in heptane) afforded $\mathbf{3 f a}$ as a white powder (30 $\mathrm{mg}, 53 \%)$. ${ }^{1} \mathrm{H}$ NMR (300 $\mathrm{MHz}, \mathrm{CDCl}_{3}$ ): $\delta=1.41(\mathrm{t}, J=7.0 \mathrm{~Hz}, 3 \mathrm{H}), 4.03(\mathrm{q}, J=7.0 \mathrm{~Hz}, 2 \mathrm{H}), 6.84$ $6.92(\mathrm{~m}, 3 \mathrm{H}), 6.97(\mathrm{~d}, J=3.9 \mathrm{~Hz}, 1 \mathrm{H}), 7.40(\mathrm{~d}, J=9.0 \mathrm{~Hz}, 2 \mathrm{H}) ;{ }^{13} \mathrm{C}$ NMR (75 MHz, $\left.\mathrm{CDCl}_{3}\right): \delta=15.0\left(\mathrm{CH}_{3}\right), 63.8\left(\mathrm{CH}_{2}\right), 110.3(\mathrm{C}), 115.2(2 \mathrm{CH}), 122.3$ $(\mathrm{CH}), 126.6(\mathrm{C}), 127.2(2 \mathrm{CH}), 130.9(\mathrm{CH}), 146.1(\mathrm{C}), 159.1(\mathrm{C})$; IR (neat) $v=2997,2896,1618,1400,1254,1164,1015 \mathrm{~cm}^{-1}$; HRMS (APPI): calc. for $\mathrm{C}_{12} \mathrm{H}_{11}{ }^{79} \mathrm{BrOS}\left[\mathrm{M}^{+}\right] \mathrm{m} / \mathrm{z} 281.9714$, found $\mathrm{m} / \mathrm{z}$ 281.9732, calc. for $\mathrm{C}_{12} \mathrm{H}_{11}{ }^{81} \mathrm{BrOS}\left[\mathrm{M}^{+}\right] \mathrm{m} / \mathrm{z} 283.9694$, found $\mathrm{m} / \mathrm{z} 283.9711$.

2-Bromo-5-(4-methylphenyl)thiophene (3fb): Prepared according to general procedure A by reacting 2,5-dibromothiophene ( $23 \mu \mathrm{L}, 0.2 \mathrm{mmol})$, with potassium 4-methylphenyltrifluoroborate $(60 \mathrm{mg}, 0.3 \mathrm{mmol})$, sodium carbonate $(85 \mathrm{mg}, 0.8 \mathrm{mmol})$, potassium chloride $(7 \mathrm{mg}, 0.1 \mathrm{mmol}$ ), triphenylphosphine $\quad(3.0 \quad \mathrm{mg}, \quad 6 \quad \mathrm{~mol} \%) \quad$ and tetrakis(triphenylphosphine)palladium( $(0)(7.0 \mathrm{mg}, 3 \mathrm{~mol} \%)$ in a mixture of degassed toluene/ethanol $(1.40 / 0.35 \mathrm{~mL})$ and degassed water $(0.70 \mathrm{~mL})$. The reaction was stirred at $70{ }^{\circ} \mathrm{C}$ overnight. After extraction, purification of the crude material by preparative TLC (100\% heptane) afforded $\mathbf{3 f b}$ as a white powder (23 mg, 45\%). ${ }^{1} \mathrm{H} \mathrm{NMR}\left(300 \mathrm{MHz}, \mathrm{CDCl}_{3}\right.$ ): $\delta=2.35$ (s, 3H), 7.00 (bs, $2 \mathrm{H}), 7.16(\mathrm{~d}, J=8.1 \mathrm{~Hz}, 2 \mathrm{H}), 7.39(\mathrm{~d}, J=8.1 \mathrm{~Hz}, 2 \mathrm{H}) ;{ }^{13} \mathrm{C}$ NMR $(75$ $\left.\mathrm{MHz}, \mathrm{CDCl}_{3}\right): \delta=21.4\left(\mathrm{CH}_{3}\right), 110.9(\mathrm{C}), 122.9(\mathrm{CH}), 125.7(2 \mathrm{CH}), 129.9$ $(2 \mathrm{CH}), 130.9(\mathrm{CH}), 131.1(\mathrm{C}), 138.1(\mathrm{C}), 146.3(\mathrm{C})$; IR (neat): $v=2994$, 1510, 1441, 1394, 1074, $1045 \mathrm{~cm}^{-1}$; HRMS (APPI): calc. for $\mathrm{C}_{11} \mathrm{H}_{9}{ }^{79} \mathrm{BrS}$ $\left[\mathrm{M}^{+}\right] \mathrm{m} / \mathrm{z} 251.9608$, found $\mathrm{m} / \mathrm{z} 251.9637$, calc. for $\mathrm{C}_{11} \mathrm{H}_{9}{ }^{81} \mathrm{BrS}\left[\mathrm{M}^{+}\right] \mathrm{m} / \mathrm{z}$ 253.9598, found $\mathrm{m} / \mathrm{z} 253.9616$.

2-Bromo-5-(4-fluorophenyl)thiophene (3fd): Prepared according to general procedure A by reacting 2,5-dibromothiophene ( $23 \mu \mathrm{L}, 0.2 \mathrm{mmol})$, with potassium 4-fluorophenyltrifluoroborate $(40 \mathrm{mg}, 0.2 \mathrm{mmol})$, sodium carbonate $(85 \mathrm{mg}, 0.8 \mathrm{mmol})$, potassium chloride $(7 \mathrm{mg}, 0.1 \mathrm{mmol}$ ), triphenylphosphine $\quad(3.0 \quad \mathrm{mg}, \quad 6 \quad \mathrm{~mol} \quad \%) \quad$ and tetrakis(triphenylphosphine)palladium( 0$)(7.0 \mathrm{mg}, 3 \mathrm{~mol} \%)$ in a mixture of degassed toluene/ethanol $(1.40 / 0.35 \mathrm{~mL})$ and degassed water $(0.70 \mathrm{~mL})$. The reaction was stirred at $70{ }^{\circ} \mathrm{C}$ overnight. After extraction, purification of the crude material by preparative TLC ( $2 \%$ MTBE in heptane) afforded $3 \mathbf{3 f}$ as a white powder (23 mg, 45\%). ${ }^{1} \mathrm{H} \mathrm{NMR}\left(300 \mathrm{MHz}, \mathrm{CDCl}_{3}\right): \delta=6.89-7.19(\mathrm{~m}$ $4 \mathrm{H}), 7.39-7.60(\mathrm{~m}, 2 \mathrm{H}) ;{ }^{13} \mathrm{C} \mathrm{NMR}\left(75 \mathrm{MHz}, \mathrm{CDCl}_{3}\right): \delta=111.3(\mathrm{C}), 116.1(\mathrm{~d}$, $J=21.8 \mathrm{~Hz}, 2 \mathrm{CH}), 123.5(\mathrm{CH}), 127.4(\mathrm{~d}, J=8.1 \mathrm{~Hz}, 2 \mathrm{CH}), 130.0(\mathrm{~d}, J=3.2$ $\mathrm{Hz}, \mathrm{C}), 131.1(\mathrm{CH}), 145.0(\mathrm{C}), 162.5$ (d, $J=246.5 \mathrm{~Hz}, \mathrm{C})$; IR (neat): $\square=$ 3082, 1613, 1381, 1394, 1170, 1037, $865 \mathrm{~cm}^{-1}$; HRMS (APPI): calc. for $\mathrm{C}_{10} \mathrm{H}_{6}{ }^{79} \mathrm{BrFS}\left[\mathrm{M}^{+}\right] \mathrm{m} / \mathrm{z} 255.9358$, found $\mathrm{m} / \mathrm{z} 255.9386$, calc. for $\mathrm{C}_{10} \mathrm{H}_{6}{ }^{81} \mathrm{BrFS}$ $\left[\mathrm{M}^{+}\right] \mathrm{m} / \mathrm{z} 257.9364$, found $\mathrm{m} / \mathrm{z} 257.9337$.

1-Trifluoromethanesulfonyl-4-(4-ethoxyphenyl)-benzene (7aa): Prepared according to general procedure A by reacting 1,4ditrifluoromethanesulfonyl-benzene $(75 \mathrm{mg}, 0.2 \mathrm{mmol}$ ), with potassium 4 ethoxyphenyltrifluoroborate ( $46 \mathrm{mg}, 0.2 \mathrm{mmol}$ ), sodium carbonate $(85 \mathrm{mg}$, $0.8 \mathrm{mmol})$, potassium chloride $(7 \mathrm{mg}, 0.1 \mathrm{mmol})$, triphenylphosphine $(3.0$ $\mathrm{mg}, 6 \mathrm{~mol} \%)$ and tetrakis(triphenylphosphine)palladium(0) $(7.0 \mathrm{mg}, 3$ mol \%) in a mixture of degassed toluene/ethanol $(1.40 / 0.35 \mathrm{~mL})$ and degassed water $(0.70 \mathrm{~mL})$. The reaction was stirred at $40{ }^{\circ} \mathrm{C}$ for 6 hours After extraction, purification of the crude material by preparative TLC (20\% $\mathrm{CH}_{2} \mathrm{Cl}_{2}$ in heptane) afforded $7 \mathbf{a a}$ as a white powder (64 mg, $\left.93 \%\right) ;{ }^{1} \mathrm{H} \mathrm{NMR}$ $\left(300 \mathrm{MHz}, \mathrm{CDCl}_{3}\right): \delta=1.43(\mathrm{t}, J=7.0 \mathrm{~Hz}, 3 \mathrm{H}), 4.07(\mathrm{q}, J=7.0 \mathrm{~Hz}, 2 \mathrm{H})$, $6.96(\mathrm{~d}, J=8.8 \mathrm{~Hz}, 2 \mathrm{H}), 7.29(\mathrm{~d}, J=8.8 \mathrm{~Hz}, 2 \mathrm{H}), 7.46(\mathrm{~d}, J=8.9 \mathrm{~Hz}, 2 \mathrm{H})$, $7.58(\mathrm{~d}, J=8.9 \mathrm{~Hz}, 2 \mathrm{H}) ;{ }^{13} \mathrm{C} \mathrm{NMR}\left(75 \mathrm{MHz}, \mathrm{CDCl}_{3}\right): \delta=15.0\left(\mathrm{CH}_{3}\right), 63.8$ $\left(\mathrm{CH}_{2}\right), 115.2(2 \mathrm{CH}), 119.1\left(\mathrm{q}, J=321.3 \mathrm{~Hz}, \mathrm{CF}_{3}\right), 121.8(2 \mathrm{CH}), 128.4(2 \mathrm{CH})$ 128.5 (2CH), 131.8 (C), $141.6(\mathrm{C}), 148.7$ (C), 159.3 (C); IR (neat): $v=2988$, 2902, 1604, 1491, 1422, 1394, 1209, 1150, 1044, $817 \mathrm{~cm}^{-1}$; HRMS (APPI) calc. for $\mathrm{C}_{15} \mathrm{H}_{13} \mathrm{~F}_{3} \mathrm{O}_{4} \mathrm{~S}\left[\mathrm{M}^{+}\right] \mathrm{m} / \mathrm{z}$ 346.0487, found $\mathrm{m} / \mathrm{z}$ 346.0469.

1-Trifluoromethanesulfonyl-4-(4-methylphenyl)-benzene (7ab): Prepared according to general procedure A by reacting 1,4ditrifluoromethanesulfonyl-benzene (75 mg, $0.2 \mathrm{mmol})$, with potassium 4 methylphenyltrifluoroborate ( $40 \mathrm{mg}, 0.2 \mathrm{mmol}$ ), sodium carbonate $(85 \mathrm{mg}$, 
$0.8 \mathrm{mmol})$, potassium chloride $(7 \mathrm{mg}, 0.1 \mathrm{mmol})$, triphenylphosphine $(3.0$ $\mathrm{mg}, 6 \mathrm{~mol} \%)$ and tetrakis(triphenylphosphine)palladium(0) $(7.0 \mathrm{mg}, 3$ mol \%) in a mixture of degassed toluene/ethanol $(1.40 / 0.35 \mathrm{~mL})$ and degassed water $(0.70 \mathrm{~mL})$. The reaction was stirred at $40{ }^{\circ} \mathrm{C}$ for 6 hours. After extraction, purification of the crude material by preparative TLC $(0 \%$ to $20 \% \mathrm{CH}_{2} \mathrm{Cl}_{2}$ in heptane) afforded $7 \mathbf{a b}$ as a white powder (44 $\mathrm{mg}, 69 \%$ ). ${ }^{1} \mathrm{H}$ NMR $\left(300 \mathrm{MHz}, \mathrm{CDCl}_{3}\right.$ ): $\delta=2.40(\mathrm{~s}, 3 \mathrm{H}), 7.24-7.27$ (m, 2H), $7.34(\mathrm{~d}, J$ $=8.8 \mathrm{~Hz}, 2 \mathrm{H}), 7.47(\mathrm{~d}, J=8.2 \mathrm{~Hz}, 2 \mathrm{H}), 7.64(\mathrm{~d}, J=8.8 \mathrm{~Hz}, 2 \mathrm{H}) ;{ }^{13} \mathrm{C} \mathrm{NMR}$ $\left(75 \mathrm{MHz}, \mathrm{CDCl}_{3}\right): \delta=21.1\left(\mathrm{CH}_{3}\right), 118.9\left(\mathrm{q}, J=321.0 \mathrm{~Hz}, \mathrm{CF}_{3}\right), 121.6(2 \mathrm{CH})$, $127.0(2 \mathrm{CH}), 128.6(2 \mathrm{CH}), 129.7(2 \mathrm{CH}), 136.4(\mathrm{C}), 138.0(\mathrm{C}), 141.6(\mathrm{C})$, 148.7 (C); IR (neat): $v=2930,1493,1425,1190,1050,810 \mathrm{~cm}^{-1}$; HRMS (APPI): calc. for $\mathrm{C}_{14} \mathrm{H}_{11} \mathrm{~F}_{3} \mathrm{O}_{3} \mathrm{~S}[\mathrm{M}+] \mathrm{m} / \mathrm{z} 316.0381$, found $\mathrm{m} / \mathrm{z} 316.0369$.

1-Trifluoromethanesulfonyl-3-(4-ethoxyphenyl)-benzene

(7ba): Prepared according to general procedure A by reacting 1,3-di trifluoromethanesulfonyl-benzene $(75 \mathrm{mg}, 0.2 \mathrm{mmol})$, with potassium 4ethoxyphenyltrifluoroborate $(46 \mathrm{mg}, 0.2 \mathrm{mmol})$, sodium carbonate $(85 \mathrm{mg}$, $0.8 \mathrm{mmol})$, potassium chloride $(7 \mathrm{mg}, 0.1 \mathrm{mmol})$, triphenylphosphine $(3.0$ $\mathrm{mg}, 6 \mathrm{~mol} \%)$ and tetrakis(triphenylphosphine)palladium $(0)(7.0 \mathrm{mg}, 3$ mol \%) in a mixture of degassed toluene/ethanol $(1.40 / 0.35 \mathrm{~mL})$ and degassed water $(0.70 \mathrm{~mL})$. The reaction was stirred at $70^{\circ} \mathrm{C}$ overnight. After extraction, purification of the crude material by preparative TLC ( $0 \%$ to $20 \%$ $\mathrm{CH}_{2} \mathrm{Cl}_{2}$ in heptane) afforded $7 \mathbf{b a}$ as a white powder $(54 \mathrm{mg}, 78 \%) .{ }^{1} \mathrm{H} \mathrm{NMR}$ $\left(300 \mathrm{MHz}, \mathrm{CDCl}_{3}\right): \delta=1.43(\mathrm{t}, J=7.0 \mathrm{~Hz}, 3 \mathrm{H}), 4.07(\mathrm{q}, J=7.0 \mathrm{~Hz}, 2 \mathrm{H})$, $6.97(\mathrm{~d}, J=8.9 \mathrm{~Hz}, 2 \mathrm{H}), 7.18(\mathrm{ddd}, J=8.0,2.3,1.0 \mathrm{~Hz}, 1 \mathrm{H}), 7.40-7.44(\mathrm{~m}$, $1 \mathrm{H}), 7.48(\mathrm{~d}, J=8.9 \mathrm{~Hz}, 2 \mathrm{H}), 7.44-7.49(\mathrm{~m}, 1 \mathrm{H}), 7.52-7.58(\mathrm{~m}, 1 \mathrm{H}) ;{ }^{13} \mathrm{C}$ $\operatorname{NMR}\left(75 \mathrm{MHz}, \mathrm{CDCl}_{3}\right): \delta=15.0\left(\mathrm{CH}_{3}\right), 63.8\left(\mathrm{CH}_{2}\right), 115.2(2 \mathrm{CH}), 119.0(\mathrm{q}$, $\left.J=320.3 \mathrm{~Hz}, \mathrm{CF}_{3}\right), 119.3(\mathrm{CH}), 119.6(\mathrm{CH}), 126.7(\mathrm{CH}), 128.4(2 \mathrm{CH}), 130.6$ (CH), 131.5 (C), 143.8 (C), 150.3 (C), 159.5 (C); IR (neat): $v=2988,2902$, 1604, 1491, 1422, 1394, 1209, 1150, 1044, $817 \mathrm{~cm}^{-1}$; HRMS (APPI): calc. for $\mathrm{C}_{15} \mathrm{H}_{13} \mathrm{~F}_{3} \mathrm{O}_{4} \mathrm{~S}\left[\mathrm{M}^{+}\right] \mathrm{m} / \mathrm{z} 346.0487$, found $\mathrm{m} / \mathrm{z} 346.0473$.

1-Trifluoromethanesulfonyl-3-(4-methylphenyl)-benzene (7bb): Prepared according to general procedure $\mathrm{A}$ by reacting $1,3-$ ditrifluoromethanesulfonyl-benzene $(75 \mathrm{mg}, 0.2 \mathrm{mmol})$, with potassium 4methylphenyltrifluoroborate $(40 \mathrm{mg}, 0.2 \mathrm{mmol})$, sodium carbonate $(85 \mathrm{mg}$, $0.8 \mathrm{mmol})$, potassium chloride $(7 \mathrm{mg}, 0.1 \mathrm{mmol})$, triphenylphosphine $(3.0$ $\mathrm{mg}, 6 \mathrm{~mol} \%)$ and tetrakis(triphenylphosphine)palladium(0) $(7.0 \mathrm{mg}, 3$ mol $\%)$ in a mixture of degassed toluene/ethanol $(1.40 / 0.35 \mathrm{~mL})$ and degassed water $(0.70 \mathrm{~mL})$. The reaction was stirred at $70^{\circ} \mathrm{C}$ overnight. After extraction, purification of the crude material by preparative TLC (20\% $\mathrm{CH}_{2} \mathrm{Cl}_{2}$ in heptane) afforded $7 \mathbf{b b}$ as a white powder (44 mg, 70\%). ${ }^{1} \mathrm{H}$ NMR $\left(300 \mathrm{MHz}, \mathrm{CDCl}_{3}\right): \delta=2.31(\mathrm{~s}, 3 \mathrm{H}), 7.09-7.16(\mathrm{~m}, 1 \mathrm{H}), 7.19(\mathrm{~d}, J=7.8 \mathrm{~Hz}$, $2 \mathrm{H}), 7.32-7.46(\mathrm{~m}, 4 \mathrm{H})$ 7.46-7.54 (m, $1 \mathrm{H}) .{ }^{13} \mathrm{C} \mathrm{NMR}\left(75 \mathrm{MHz}, \mathrm{CDCl}_{3}\right): \delta=$ $21.3\left(\mathrm{CH}_{3}\right), 119.0\left(\mathrm{q}, J=318 \mathrm{~Hz}, \mathrm{CF}_{3}\right), 119.7(\mathrm{CH}), 119.9(\mathrm{CH}), 127.0(\mathrm{CH})$, $127.2(2 \mathrm{CH}), 130.0(2 \mathrm{CH}), 130.6(\mathrm{CH}), 136.4(\mathrm{C}), 138.6(\mathrm{C}), 144.2(\mathrm{C})$, 150.3 (C); IR (neat): $v=2945,2855,1598,1530,1417,1345,1212,1126$ $\mathrm{cm}^{-1}$; HRMS (APPI): calc. for $\mathrm{C}_{14} \mathrm{H}_{11} \mathrm{~F}_{3} \mathrm{O}_{3} \mathrm{~S}[\mathrm{M}+] \mathrm{m} / \mathrm{z} 316.0381$, found $\mathrm{m} / \mathrm{z}$ 316.0372.

1-Trifluoromethanesulfonyl-3-(4-nitrophenyl)benzene

(7bc): Prepared according to general procedure A by reacting 1,3-di trifluoromethanesulfonylbenzene $(75 \mathrm{mg}, 0.2 \mathrm{mmol})$, with potassium 4nitrophenyltrifluoroborate ( $46 \mathrm{mg}, 0.2 \mathrm{mmol}$ ), sodium carbonate $(85 \mathrm{mg}, 0.8$ $\mathrm{mmol}$ ), potassium chloride ( $7 \mathrm{mg}, 0.1 \mathrm{mmol})$, triphenylphosphine $(3.0 \mathrm{mg}, 6$ mol \%) and tetrakis(triphenylphosphine)palladium( 0$)(7.0 \mathrm{mg}, 3 \mathrm{~mol} \%)$ in a mixture of degassed toluene/ethanol $(1.40 / 0.35 \mathrm{~mL})$ and degassed water $(0.70 \mathrm{~mL})$. The reaction was stirred at $70{ }^{\circ} \mathrm{C}$ overnight. After extraction, purification of the crude material by preparative TLC $\left(20 \% \mathrm{CH}_{2} \mathrm{Cl}_{2}\right.$ in heptane) afforded $\mathbf{7 b c}$ as a white powder (39 mg, 57\%). ${ }^{1} \mathrm{H} \mathrm{NMR}(300 \mathrm{MHz}$, $\left.\mathrm{CDCl}_{3}\right): \delta=7.30-7.40(\mathrm{~m}, 1 \mathrm{H}), 7.50(\mathrm{t}, J=1.9 \mathrm{~Hz}, 1 \mathrm{H}), 7.54-7.67(\mathrm{~m}, 2 \mathrm{H})$, $7.71(\mathrm{~d}, J=8.9 \mathrm{~Hz}, 2 \mathrm{H}), 8.32(\mathrm{~d}, J=8.9 \mathrm{~Hz}, 2 \mathrm{H}) ;{ }^{13} \mathrm{C} \mathrm{NMR}(75 \mathrm{MHz}$, $\left.\mathrm{CDCl}_{3}\right): \delta=119.0\left(\mathrm{q}, J=319.4 \mathrm{~Hz}, \mathrm{CF}_{3}\right), 120.6(\mathrm{CH}), 121.7(\mathrm{CH}), 124.6$ $(2 \mathrm{CH}), 127.6(\mathrm{CH}), 128.3(2 \mathrm{CH}), 131.3(\mathrm{CH}), 141.7(\mathrm{C}), 145.4(\mathrm{C}), 148.0$ (C), 150.3 (C); IR (neat): $v=2925,2865,1613,1481,1421,1244,1200$, $1139,1126 \mathrm{~cm}^{-1}$; MS: no ionization could be observed for this compound.
1-Trifluoromethanesulfonyl-2-(4-ethoxyphenyl)-benzene (7ca): Prepared according to general procedure $\mathrm{A}$ by reacting 1,2ditrifluoromethanesulfonyl-benzene ( $75 \mathrm{mg}, 0.2 \mathrm{mmol})$, with potassium 4 ethoxyphenyltrifluoroborate ( $46 \mathrm{mg}, 0.2 \mathrm{mmol})$, sodium carbonate $(85 \mathrm{mg}$, $0.8 \mathrm{mmol})$, potassium chloride $(7 \mathrm{mg}, 0.1 \mathrm{mmol})$, triphenylphosphine $(3.0$ $\mathrm{mg}, 6 \mathrm{~mol} \%)$ and tetrakis(triphenylphosphine)palladium $(0)(7.0 \mathrm{mg}, 3$ mol \%) in a mixture of degassed toluene/ethanol $(1.40 / 0.35 \mathrm{~mL})$ and degassed water $(0.70 \mathrm{~mL})$. The reaction was stirred at $70{ }^{\circ} \mathrm{C}$ overnight. After extraction, purification of the crude material by preparative TLC ( $0 \%$ to $20 \%$ $\mathrm{CH}_{2} \mathrm{Cl}_{2}$ in heptane) afforded $\mathbf{7} \mathbf{c a}$ as a white powder $(45 \mathrm{mg}, 65 \%) .{ }^{1} \mathrm{H} \mathrm{NMR}$ $\left(300 \mathrm{MHz}, \mathrm{CDCl}_{3}\right): \delta=1.36(\mathrm{t}, J=7.0 \mathrm{~Hz}, 3 \mathrm{H}), 4.00(\mathrm{q}, J=7.0 \mathrm{~Hz}, 2 \mathrm{H})$, $6.89(\mathrm{~d}, J=8.8 \mathrm{~Hz}, 2 \mathrm{H}), 7.28-7.54(\mathrm{~m}, 6 \mathrm{H}) ;{ }^{13} \mathrm{C} \mathrm{NMR}\left(75 \mathrm{MHz}, \mathrm{CDCl}_{3}\right): \delta$ $=15.0\left(\mathrm{CH}_{3}\right), 63.7\left(\mathrm{CH}_{2}\right), 114.7(2 \mathrm{CH}), 118.6\left(\mathrm{q}, J=321.2 \mathrm{~Hz}, \mathrm{CF}_{3}\right), 122.3$ $(\mathrm{CH}), 128.0(\mathrm{C}), 128.6(\mathrm{CH}), 128.7(\mathrm{CH}), 130.7(2 \mathrm{CH}), 132.1(\mathrm{CH}), 135.5$ (C), 147.1 (C), 159.3 (C); IR (neat): $v=2990,2888,1614,1495,1422,1397$, 1209, 1162, 1056, $877 \mathrm{~cm}^{-1}$; HRMS (APPI): calc. for $\mathrm{C}_{15} \mathrm{H}_{13} \mathrm{~F}_{3} \mathrm{O}_{4} \mathrm{~S}\left[\mathrm{M}^{+}\right] \mathrm{m} / \mathrm{z}$ 346.0487, found $\mathrm{m} / \mathrm{z} 346.0481$.

1-Trifluoromethanesulfonyl-2-(4-methylphenyl)-benzene (7cb): Prepared according to general procedure A by reacting 1,2ditrifluoromethanesulfonyl-benzene (75 $\mathrm{mg}, 0.2 \mathrm{mmol})$, with potassium 4 methylphenyltrifluoroborate ( $40 \mathrm{mg}, 0.2 \mathrm{mmol})$, sodium carbonate $(85 \mathrm{mg}$, $0.8 \mathrm{mmol})$, potassium chloride $(7 \mathrm{mg}, 0.1 \mathrm{mmol})$, triphenylphosphine $(3.0$ $\mathrm{mg}, 6 \mathrm{~mol} \%)$ and tetrakis(triphenylphosphine)palladium(0) $(7.0 \mathrm{mg}, 3$ mol \%) in a mixture of degassed toluene/ethanol $(1.40 / 0.35 \mathrm{~mL})$ and degassed water $(0.70 \mathrm{~mL})$. The reaction was stirred at $70^{\circ} \mathrm{C}$ overnight. After extraction, purification of the crude material by flash column chromatography on silica gel (2\% EtOAc in heptane) afforded $\mathbf{7 c b}$ as a white powder (41 mg, 62\%). ${ }^{1} \mathrm{H}$ NMR (300 MHz, $\mathrm{CDCl}_{3}$ ): $\delta=2.33$ (s, 3H), 7.18 $(\mathrm{d}, J=7.5 \mathrm{~Hz}, 2 \mathrm{H}), 7.23-7.43(\mathrm{~m}, 6 \mathrm{H}) ;{ }^{13} \mathrm{C} \mathrm{NMR}\left(75 \mathrm{MHz}, \mathrm{CDCl}_{3}\right): \delta=21.3$ $\left(\mathrm{CH}_{3}\right), 118.4\left(\mathrm{q}, J=320.2 \mathrm{~Hz}, \mathrm{CF}_{3}\right), 122.0(\mathrm{CH}), 128.5(\mathrm{CH}), 128.7(\mathrm{CH})$, $129.2(4 \mathrm{CH}), 132.0(\mathrm{CH}), 132.7(\mathrm{C}), 135.6(\mathrm{C}), 138.2(\mathrm{C}), 146.9(\mathrm{C})$; IR (neat): $v=2990,2888,1614,1495,1422,1397,1209,1162,1056,877 \mathrm{~cm}^{-1}$; HRMS (APPI): calc. for $\mathrm{C}_{14} \mathrm{H}_{11} \mathrm{~F}_{3} \mathrm{O}_{3} \mathrm{~S}\left[\mathrm{M}^{+}\right] \mathrm{m} / \mathrm{z}$ 316.0381, found $\mathrm{m} / \mathrm{z}$ 316.0372 .

1-Trifluoromethanesulfonyl-2-(4-nitrophenyl)benzene (7cc): Prepared according to general procedure $\mathrm{A}$ by reacting 1,2-ditrifluoromethanesulfonylbenzene $(75 \mathrm{mg}, 0.2 \mathrm{mmol})$, with potassium 4 nitrophenyltrifluoroborate ( $46 \mathrm{mg}, 0.2 \mathrm{mmol}$ ), sodium carbonate $(85 \mathrm{mg}, 0.8$ $\mathrm{mmol})$, potassium chloride $(7 \mathrm{mg}, 0.1 \mathrm{mmol})$, triphenylphosphine $(3.0 \mathrm{mg}, 6$ $\mathrm{mol} \%$ ) and tetrakis(triphenylphosphine)palladium $(0)(7.0 \mathrm{mg}, 3 \mathrm{~mol} \%)$ in a mixture of degassed toluene/ethanol $(1.40 / 0.35 \mathrm{~mL})$ and degassed water $(0.70 \mathrm{~mL})$. The reaction was stirred at $70{ }^{\circ} \mathrm{C}$ overnight. After extraction, purification of the crude material by preparative TLC ( $0 \%$ to $20 \% \mathrm{CH}_{2} \mathrm{Cl}_{2}$ in heptane) afforded 7cc as a white powder $(25 \mathrm{mg}, 38 \%) .{ }^{1} \mathrm{H} \mathrm{NMR}(300 \mathrm{MHz}$, $\left.\mathrm{CDCl}_{3}\right): \delta=7.39-7.56(\mathrm{~m}, 4 \mathrm{H}), 7.63(\mathrm{~d}, J=8.9 \mathrm{~Hz}, 2 \mathrm{H}), 8.31(\mathrm{~d}, J=8.9 \mathrm{~Hz}$, $2 \mathrm{H}$ ); ${ }^{13} \mathrm{C}$ NMR (75 MHz, $\left.\mathrm{CDCl}_{3}\right): \delta=118.3$ (q, $J=321.0 \mathrm{~Hz}, \mathrm{CF}_{3}$ ), 122.7 $(\mathrm{CH}), 124.0(2 \mathrm{CH}), 129.1(\mathrm{CH}), 130.6(2 \mathrm{CH}), 130.7(\mathrm{CH}), 131.9(\mathrm{CH}), 133.6$ (C), 142.4 (C), 146.6 (C), 147.9 (C); IR (neat): $v=2922,2871,1613,1487$, $1415,1249,1212,1143,1128 \mathrm{~cm}^{-1}$; MS: no ionization could be observed for this compound.

Diethyl- $N$-benzyl-3-(4-ethoxyphenyl)-4-triflyl-pyrrole-2,5carboxylate (7da): Prepared according to general procedure A by reacting pyrrole 6d (120 mg, $0.2 \mathrm{mmol})$, with potassium 4ethoxyphenyltrifluoroborate ( $46 \mathrm{mg}, 0.2 \mathrm{mmol})$, sodium carbonate $(85 \mathrm{mg}$, $0.8 \mathrm{mmol}$ ), potassium chloride $(7 \mathrm{mg}, 0.1 \mathrm{mmol})$, triphenylphosphine (3.0 $\mathrm{mg}, 6 \mathrm{~mol} \%)$ and tetrakis(triphenylphosphine)palladium(0) $(7.0 \mathrm{mg}, 3$ mol \%) in a mixture of degassed toluene/ethanol $(1.40 / 0.35 \mathrm{~mL})$ and degassed water $(0.70 \mathrm{~mL})$. The reaction was stirred at $70^{\circ} \mathrm{C}$ overnight. After extraction, purification of the crude material by flash column chromatography on neutral alumina gel ( $2 \%$ EtOAc in heptane) afforded 7da as a white powder (58 mg, 51\%). ${ }^{1} \mathrm{H} \mathrm{NMR}\left(300 \mathrm{MHz}, \mathrm{CDCl}_{3}\right): \delta=0.93(\mathrm{t}, J$ $=6.9 \mathrm{~Hz}, 3 \mathrm{H}), 1.32(\mathrm{t}, J=7.2 \mathrm{~Hz}, 3 \mathrm{H}), 1.41(\mathrm{t}, J=7.2 \mathrm{~Hz}, 3 \mathrm{H}), 3.98-4.09$ $(\mathrm{m}, 4 \mathrm{H}), 4.34(\mathrm{q}, J=6.9 \mathrm{~Hz}, 2 \mathrm{H}), 6.08(\mathrm{~s}, 2 \mathrm{H}), 6.88(\mathrm{~d}, J=8.7 \mathrm{~Hz}, 2 \mathrm{H}), 7.00$ 
$(\mathrm{d}, J=6.6 \mathrm{~Hz}, 2 \mathrm{H}), 7.16-7.31(\mathrm{~m}, 5 \mathrm{H}) ;{ }^{13} \mathrm{C} \mathrm{NMR}\left(125 \mathrm{MHz}, \mathrm{CDCl}_{3}\right): \delta=$ 13.6 $\left(\mathrm{CH}_{3}\right), 13.9\left(\mathrm{CH}_{3}\right), 14.8\left(\mathrm{CH}_{3}\right), 49.8\left(\mathrm{CH}_{2}\right), 61.2\left(\mathrm{CH}_{2}\right), 61.7\left(\mathrm{CH}_{2}\right), 63.5$ $\left(\mathrm{CH}_{2}\right), 113.9(2 \mathrm{CH}), 118.0\left(\mathrm{q}, J=317.0 \mathrm{~Hz}, \mathrm{CF}_{3}\right), 118.3(\mathrm{C}), 121.9(\mathrm{C}), 123.3$ $(2 \mathrm{C}), 126.2(2 \mathrm{CH}), 127.3(\mathrm{CH}), 128.4(2 \mathrm{CH}), 131.5(2 \mathrm{CH}), 136.1(\mathrm{C}), 137.7$ (C), 158.9 (C), 159.1 (C), 160.6 (C); IR (neat): $v=2983,1721,1556,1425$, 1286, 1243, 1185, 1135, 1022, 921, 829, $731 \mathrm{~cm}^{-1}$; HRMS (ESI): $\mathrm{m} / z=$ 570.1433, calcd. for $[\mathrm{M}+\mathrm{H}]^{+} \mathrm{C}_{26} \mathrm{H}_{27} \mathrm{~F}_{3} \mathrm{NO}_{8} \mathrm{~S}: 570.1409$.

Diethyl- $N$-benzyl-3-(4-methylphenyl)-4-triflyl-pyrrole-2,5carboxylate (7db): Prepared according to general procedure A by reacting pyrrole 6d $(120 \quad \mathrm{mg}, \quad 0.2 \mathrm{mmol})$, with potassium 4methylphenyltrifluoroborate $(40 \mathrm{mg}, 0.2 \mathrm{mmol})$, sodium carbonate $(85 \mathrm{mg}$, $0.8 \mathrm{mmol})$, potassium chloride $(7 \mathrm{mg}, 0.1 \mathrm{mmol})$, triphenylphosphine $(3.0$ $\mathrm{mg}, 6 \mathrm{~mol} \%)$ and tetrakis(triphenylphosphine)palladium(0) $(7.0 \mathrm{mg}, 3$ mol $\%)$ in a mixture of degassed toluene/ethanol $(1.40 / 0.35 \mathrm{~mL})$ and degassed water $(0.70 \mathrm{~mL})$. The reaction was stirred at $70^{\circ} \mathrm{C}$ overnight. After extraction, purification of the crude material by flash column chromatography on neutral alumina gel $\left(30 \% \mathrm{CH}_{2} \mathrm{Cl}_{2}\right.$ in heptane) afforded $7 \mathbf{d b}$ as a white powder $(62 \mathrm{mg}, 57 \%) .{ }^{1} \mathrm{H}$ NMR $\left(75 \mathrm{MHz}, \mathrm{CDCl}_{3}\right): \delta=0.85$ (t, $J=7.2 \mathrm{~Hz}, 3 \mathrm{H}), 1.27$ (t, $J=7.2 \mathrm{~Hz}, 3 \mathrm{H}), 2.31$ (s, 3H), 3.96 (q, $J=7.2 \mathrm{~Hz}$, $2 \mathrm{H}), 4.28(\mathrm{q}, J=7.2 \mathrm{~Hz}, 2 \mathrm{H}), 6.02(\mathrm{~s}, 2 \mathrm{H}), 6.95(\mathrm{~d}, J=6.9 \mathrm{~Hz}, 2 \mathrm{H}), 7.06-$ $7.28(\mathrm{~m}, 7 \mathrm{H}) ;{ }^{13} \mathrm{C} \mathrm{NMR}\left(75 \mathrm{MHz}, \mathrm{CDCl}_{3}\right): \delta=13.5\left(\mathrm{CH}_{3}\right), 14.0\left(\mathrm{CH}_{3}\right), 21.3$ $\left(\mathrm{CH}_{3}\right), 49.6\left(\mathrm{CH}_{2}\right), 61.3\left(\mathrm{CH}_{2}\right), 61.8\left(\mathrm{CH}_{2}\right), 118.0\left(\mathrm{q}, J=318.0 \mathrm{~Hz}, \mathrm{CF}_{3}\right)$, $118.3(\mathrm{C}), 123.4(\mathrm{C}), 123.5(\mathrm{C}), 126.2(2 \mathrm{CH}), 126.7(\mathrm{C}), 127.4(\mathrm{CH}), 128.5$ $(2 \mathrm{CH}), 128.6(2 \mathrm{CH}), 130.1(2 \mathrm{CH}), 136.0(\mathrm{C}), 137.7(\mathrm{C}), 137.8(\mathrm{C}), 159.1$ (C), 160.6 (C); IR (neat): $v=2963,1718,1424,1289,1220,1187,1133$, 1021, 925, 820, $697 \mathrm{~cm}^{-1}$; HRMS (ESI): $\mathrm{m} / z=540.1299$, calcd. for $[\mathrm{M}+\mathrm{H}]^{+}$ $\mathrm{C}_{25} \mathrm{H}_{25} \mathrm{~F}_{3} \mathrm{NO}_{7} \mathrm{~S}: 540.1304$.

Diethyl- $N$-benzyl-3-(4-nitrophenyl)-4-triflyl-pyrrole-2,5-carboxylate (7dc): Prepared according to general procedure A by reacting pyrrole $\mathbf{6 d}(60$ $\mathrm{mg}, 0.1 \mathrm{mmol}$ ), with potassium 4-nitrophenyltrifluoroborate $(23 \mathrm{mg}, 0.1$ $\mathrm{mmol}$ ), sodium carbonate ( $43 \mathrm{mg}, 0.4 \mathrm{mmol}$ ), potassium chloride $(7 \mathrm{mg}, 0.1$ mmol), triphenylphosphine $(1.5 \quad \mathrm{mg}, \quad 3 \quad \mathrm{~mol} \%) \quad$ and tetrakis(triphenylphosphine)palladium( $(0)(3.5 \mathrm{mg}, 1.5 \mathrm{~mol} \%)$ in a mixture of degassed toluene/ethanol $(0.70 / 0.20 \mathrm{~mL})$ and degassed water $(0.35 \mathrm{~mL})$. The reaction was stirred at $70{ }^{\circ} \mathrm{C}$ overnight. After extraction, purification of the crude material by flash column chromatography on silica gel $(10 \%$ EtOAc in heptane) afforded $\mathbf{7 d c}$ as a white powder $(17 \mathrm{mg}, 31 \%) .{ }^{1} \mathrm{H}$ NMR $\left(300 \mathrm{MHz}, \mathrm{CDCl}_{3}\right): \delta=0.85(\mathrm{t}, J=7.2 \mathrm{~Hz}, 3 \mathrm{H}), 1.28(\mathrm{t}, J=7.2 \mathrm{~Hz}, 3 \mathrm{H}), 3.98$ $(\mathrm{q}, J=7.2 \mathrm{~Hz}, 2 \mathrm{H}), 4.30(\mathrm{q}, J=7.2 \mathrm{~Hz}, 2 \mathrm{H}), 6.08(\mathrm{~s}, 2 \mathrm{H}), 6.96(\mathrm{~d}, J=6.9$ $\mathrm{Hz}, 2 \mathrm{H}), 7.17-7.31(\mathrm{~m}, 3 \mathrm{H}), 7.44(\mathrm{~d}, J=8.7 \mathrm{~Hz}, 2 \mathrm{H}), 8.19(\mathrm{~d}, J=8.7 \mathrm{~Hz}$, $2 \mathrm{H}) ;{ }^{13} \mathrm{C}$ NMR $(75 \mathrm{MHz}, \mathrm{CDCl}): \delta=13.5\left(\mathrm{CH}_{3}\right), 13.9\left(\mathrm{CH}_{3}\right), 49.9\left(\mathrm{CH}_{2}\right)$, $61.7\left(\mathrm{CH}_{2}\right), 62.2\left(\mathrm{CH}_{2}\right), 117.9\left(\mathrm{q}, J=319.5 \mathrm{~Hz}, \mathrm{CF}_{3}\right), 119.1(\mathrm{C}), 121.2(\mathrm{C})$, $123.0(2 \mathrm{CH}), 123.2(\mathrm{C}), 126.3(2 \mathrm{CH}), 127.6(\mathrm{CH}), 128.7(2 \mathrm{CH}), 131.4(2 \mathrm{CH})$ 135.4 (C), 137.1 (C), 137.2 (C), 147.5 (C), 158.9 (C), 159.8 (C); IR (neat): v $=2919,1726,1524,1421,1348,1276,1216,1187,1133,1018,930,853$, $760 \mathrm{~cm}^{-1}$; HRMS (ESI): $m / z=571.0973$, calcd. for $[\mathrm{M}+\mathrm{H}]^{+} \mathrm{C}_{24} \mathrm{H}_{22} \mathrm{~F}_{3} \mathrm{~N}_{2} \mathrm{O}_{9} \mathrm{~S}$ : 571.0998 .

2-(4-Fluorophenyl)-6-(4-ethoxyphenyl)pyridine (8): $\quad$ Prepared according to general procedure B by reacting 2,6-dibromopyridine (46 mg, $0.2 \mathrm{mmol}$ ), with potassium 4-ethoxyphenyltrifluoroborate $(46 \mathrm{mg}, 0.2 \mathrm{mmol}$ ), sodium carbonate ( $170 \mathrm{mg}, 1.6 \mathrm{mmol})$, potassium chloride $(7 \mathrm{mg}, 0.1 \mathrm{mmol})$, triphenylphosphine $\quad(3.0 \quad \mathrm{mg}, \quad 6 \quad \mathrm{~mol} \quad \%) \quad$ and tetrakis(triphenylphosphine)palladium( 0$)(7.0 \mathrm{mg}, 3 \mathrm{~mol} \%)$ in a mixture of degassed toluene/ethanol $(1.40 / 0.35 \mathrm{~mL})$ and degassed water $(0.70 \mathrm{~mL})$. The reaction was first stirred at $40{ }^{\circ} \mathrm{C}$ for $6 \mathrm{~h}$. Potassium 4fluorophenyltrifluoroborate $(40 \mathrm{mg}, 0.2 \mathrm{mmol}$ ) was then added at $\mathrm{rt}$ and the mixture was heated at $100{ }^{\circ} \mathrm{C}$ during $2 \mathrm{~h}$. After filtration on silica, chromatography of the crude reaction product on silica using $0 \%$ to $20 \%$ EtOAc in heptane afforded 8 (49.0 mg, 83\%). $\left.{ }^{1} \mathrm{H} \mathrm{NMR} \mathrm{(300} \mathrm{MHz,} \mathrm{CDCl}_{3}\right)$ : $\delta=1.37(\mathrm{t}, J=7.1 \mathrm{~Hz}, 3 \mathrm{H}), 4.02(\mathrm{q}, J=7.1 \mathrm{~Hz}, 2 \mathrm{H}), 6.92(\mathrm{~d}, J=8.9 \mathrm{~Hz}, 2 \mathrm{H})$, $7.09(\mathrm{t}, J=8.1 \mathrm{~Hz}, 2 \mathrm{H}), 7.55-7.48(\mathrm{~m}, 2 \mathrm{H}), 7.68(\mathrm{t}, J=8.1 \mathrm{~Hz}, 1 \mathrm{H}), 8.00(\mathrm{~d}$, $J=8.9 \mathrm{~Hz}, 2 \mathrm{H}), 8.08-8.03(\mathrm{~m}, 2 \mathrm{H}) ;{ }^{13} \mathrm{C}$ NMR $\left(75 \mathrm{MHz}, \mathrm{CDCl}_{3}\right): \delta=14.8$ $\left(\mathrm{CH}_{3}\right), 63.6\left(\mathrm{CH}_{2}\right), 114.6(2 \mathrm{CH}), 115.5(\mathrm{~d}, J=21.2 \mathrm{~Hz}, 2 \mathrm{CH}), 117.5(\mathrm{CH})$,
$117.8(\mathrm{CH}), 128.2(2 \mathrm{CH}), 128.7(\mathrm{~d}, J=8.3 \mathrm{~Hz}, 2 \mathrm{CH}), 131.8(\mathrm{C}), 135.8(\mathrm{~d}, J$ $=2.8 \mathrm{~Hz}, \mathrm{C}), 137.4(\mathrm{CH}), 155.6(\mathrm{C}), 156.6(\mathrm{C}), 159.9(\mathrm{C}), 163.5(\mathrm{~d}, J=246.5$ $\mathrm{Hz}, \mathrm{C})$; IR (neat): $v=2940,1515,1413,1344,701 \mathrm{~cm}^{-1}$; HRMS (ESI): calc. for $\mathrm{C}_{19} \mathrm{H}_{17} \mathrm{FNO}[\mathrm{M}+\mathrm{H}]^{+} \mathrm{m} / \mathrm{z} 294.1289$, found $\mathrm{m} / \mathrm{z} 294.1301$.

4-Ethoxy-4"'-(trifluoromethyl)-1,1':3',1'-terphenyl (9): Prepared according to the general procedure B by reacting 1,3-dibromobenzene 1c (24 $\mu \mathrm{L}, 0.2 \mathrm{mmol}$ ), with potassium 4-ethoxyphenyltrifluoroborate ( $46 \mathrm{mg}, 0.2$ $\mathrm{mmol}$ ), sodium carbonate $(170 \mathrm{mg}, 1.6 \mathrm{mmol})$, potassium chloride $(7 \mathrm{mg}, 0.1$ $\mathrm{mmol})$ triphenylphosphine $(3.0 \quad \mathrm{mg}, \quad 6 \quad \mathrm{~mol} \%) \quad$ and tetrakis(triphenylphosphine)palladium( $(0)(7.0 \mathrm{mg}, 3 \mathrm{~mol} \%)$ in a mixture of degassed toluene/ethanol $(1.40 / 0.35 \mathrm{~mL})$ and degassed water $(0.70 \mathrm{~mL})$. The reaction was stirred at $40{ }^{\circ} \mathrm{C}$ for 6 hours. 4-trifluoromethylphenylboronic acid $\left(76 \mathrm{mg}, 0.4 \mathrm{mmol}\right.$ ) was then added and the mixture was heated at $70{ }^{\circ} \mathrm{C}$ during $20 \mathrm{~h}$. After extraction, purification of the crude material by flash column chromatography on silica gel $\left(2 \% \mathrm{CH}_{2} \mathrm{Cl}_{2}\right.$ in heptane) afforded $\mathbf{9}$ as a white powder (39 mg, $58 \%$ ).

Prepared according to the general procedure B by reacting 1,3ditrifluoromethanesulfonylbenzene $6 \mathbf{b}$ ( $75 \mathrm{mg}, 0.2 \mathrm{mmol}$ ), with potassium 4ethoxyphenyltrifluoroborate ( $46 \mathrm{mg}, 0.2 \mathrm{mmol}$ ), sodium carbonate (170 mg, $1.6 \mathrm{mmol}$ ), potassium chloride $(7 \mathrm{mg}, 0.1 \mathrm{mmol})$, triphenylphosphine (3.0 $\mathrm{mg}, 6 \mathrm{~mol} \%)$ and tetrakis(triphenylphosphine)palladium $(0)(7.0 \mathrm{mg}, 3$ $\mathrm{mol} \%)$ in a mixture of degassed toluene/ethanol $(1.40 / 0.35 \mathrm{~mL})$ and degassed water $(0.70 \mathrm{~mL})$. The reaction was first stirred at $70{ }^{\circ} \mathrm{C}$ for $20 \mathrm{~h} .4-$ Trifluoromethylphenylboronic acid ( $38 \mathrm{mg}, 0.2 \mathrm{mmol}$ ) was then added and the mixture was heated at $70{ }^{\circ} \mathrm{C}$ during $20 \mathrm{~h}$. After extraction, purification of the crude material by preparative TLC ( $2 \% \mathrm{CH}_{2} \mathrm{Cl}_{2}$ in heptane) afforded 9 as a white powder $(42 \mathrm{mg}, 62 \%) .{ }^{1} \mathrm{H} \mathrm{NMR}\left(300 \mathrm{MHz}, \mathrm{CDCl}_{3}\right): \delta=1.43(\mathrm{t}, J$ $=7.0 \mathrm{~Hz}, 3 \mathrm{H}), 4.08(\mathrm{q}, J=7.0 \mathrm{~Hz}, 2 \mathrm{H}), 6.98(\mathrm{~d}, J=8.7 \mathrm{~Hz}, 2 \mathrm{H}), 7.47-7.61$ $(\mathrm{m}, 5 \mathrm{H}), 7.66-7.79(\mathrm{~m}, 5 \mathrm{H}) ;{ }^{13} \mathrm{C} \mathrm{NMR}\left(75 \mathrm{MHz}, \mathrm{CDCl}_{3}\right): \delta=15.1\left(\mathrm{CH}_{3}\right)$, $63.8\left(\mathrm{CH}_{2}\right), 115.1(2 \mathrm{CH}), 124.5\left(\mathrm{q}, J=271.8 \mathrm{~Hz}, \mathrm{CF}_{3}\right), 125.8(\mathrm{CH}), 125.9(\mathrm{q}$, $J=3.6 \mathrm{~Hz}, 2 \mathrm{CH}), 126.0(\mathrm{CH}), 126.8(\mathrm{CH}), 127.7(2 \mathrm{CH}), 128.5(2 \mathrm{CH}), 129.6$ $(\mathrm{CH}), 129.7$ (q, $J=32.0 \mathrm{~Hz}, \mathrm{C}), 133.4(\mathrm{C}), 140.5$ (C), $141.9(\mathrm{C}), 145.0(\mathrm{C})$, 159.0 (C); IR (neat): $v=2987,2926,1605,1516,1475,1328,1252,1170$, $1129 \mathrm{~cm}^{-1}$; HRMS (ESI): $\mathrm{m} / z=425.2329$, calcd. for $[\mathrm{M}+2 \mathrm{MeCN}+\mathrm{H}]^{+}$ $\mathrm{C}_{25} \mathrm{H}_{24} \mathrm{~F}_{3} \mathrm{~N}_{2} \mathrm{O}: 425.1841$.

Diethyl- $N$-benzyl-3-(2-benzyloxyphenyl)-4-(3,4-dimethoxyphenyl)pyrrole-2,5-carboxylate (10): Prepared according to general procedure B by reacting pyrrole $\mathbf{6 d}(60 \mathrm{mg}, 0.1 \mathrm{mmol})$, with potassium 3,4dimethoxyphenyltrifluoroborate $(25 \mathrm{mg}, 0.1 \mathrm{mmol})$, sodium carbonate ( 85 $\mathrm{mg}, 0.8 \mathrm{mmol})$, potassium chloride ( $3.5 \mathrm{mg}, 0.05 \mathrm{mmol})$, triphenylphosphine $(1.5 \mathrm{mg}, 6 \mathrm{~mol} \%)$ and tetrakis(triphenylphosphine)palladium(0) $(3.5 \mathrm{mg}, 3$ $\mathrm{mol} \%)$ in a mixture of degassed toluene/ethanol $(0.7 / 0.2 \mathrm{~mL})$ and degassed water $(0.35 \mathrm{~mL})$. The reaction was first stirred at $70{ }^{\circ} \mathrm{C}$ for $20 \mathrm{~h} .2$ Benzyloxyphenyboronic acid $(27 \mathrm{mg}, 0.12 \mathrm{mmol})$ was then added at $\mathrm{rt}$ and the mixture was heated at $100{ }^{\circ} \mathrm{C}$ during $20 \mathrm{~h}$. After filtration on silica, chromatography of the crude reaction product on silica using $0 \%$ to $20 \%$ EtOAc in heptane afforded $\mathbf{1 0}$ as a white powder $(47.0 \mathrm{mg}, 76 \%) .{ }^{1} \mathrm{H}$ NMR $\left(300 \mathrm{MHz}, \mathrm{CDCl}_{3}\right): \delta=0.68(\mathrm{t}, J=6.8 \mathrm{~Hz}, 3 \mathrm{H}), 0.83(\mathrm{t}, J=6.8 \mathrm{~Hz}, 3 \mathrm{H}), 3.43$ (s, 3H), 3.72 (s, 3H), $3.86(\mathrm{q}, J=6.8 \mathrm{~Hz}, 2 \mathrm{H}), 3.94$ (q, $J=6.8 \mathrm{~Hz}, 2 \mathrm{H}), 4.84$ $(\mathrm{s}, 2 \mathrm{H}), 6.03(\mathrm{~s}, 2 \mathrm{H}), 6.50(\mathrm{~s}, 1 \mathrm{H}), 6.57(\mathrm{~s}, 1 \mathrm{H}), 6.58(\mathrm{~s}, 1 \mathrm{H}), 6.74(\mathrm{~d}, J=7.4$ $\mathrm{Hz}, 2 \mathrm{H}), 6.90(\mathrm{dd}, J=7.4,1.2 \mathrm{~Hz}, 1 \mathrm{H}), 7.05-6.98(\mathrm{~m}, 2 \mathrm{H}), 7.28-7.06(\mathrm{~m}$, 9H); $\left.{ }^{13} \mathrm{C} \mathrm{NMR} \mathrm{(75} \mathrm{MHz,} \mathrm{CDCl} 3\right): \delta=13.3\left(\mathrm{CH}_{3}\right), 13.6\left(\mathrm{CH}_{3}\right), 49.6\left(\mathrm{CH}_{2}\right)$, $55.4\left(\mathrm{CH}_{3}\right), 55.7\left(\mathrm{CH}_{3}\right), 60.2\left(\mathrm{CH}_{2}\right), 60.6\left(\mathrm{CH}_{2}\right), 69.8\left(\mathrm{CH}_{2}\right), 109.9(\mathrm{CH})$, $111.9(\mathrm{CH}), 113.5(\mathrm{CH}), 120.3(\mathrm{CH}), 122.6(\mathrm{CH}), 124.8(\mathrm{C}), 124.9(\mathrm{C}), 125.2$ (C), $126.2(2 \mathrm{CH}), 126.7(2 \mathrm{CH}), 126.8(\mathrm{CH}), 127.2(\mathrm{C}), 127.5(\mathrm{CH}), 127.8$ (C), $128.2(\mathrm{CH}), 128.4(2 \mathrm{CH}), 128.5(2 \mathrm{CH}), 131.2(\mathrm{C}), 132.0(\mathrm{CH}), 137.4$ (C), 139.1 (C), 147.4 (C), 147.5 (C), 156.4 (C), 161.4 (C), 161.8 (C); IR (neat): $v=2927,2853,1712,1454,1234,1200,1138,1027,731 \mathrm{~cm}^{-1} ;$ HRMS (ESI): calc. for $\mathrm{C}_{38} \mathrm{H}_{38} \mathrm{NO}_{7}[\mathrm{M}+\mathrm{H}]^{+} \mathrm{m} / \mathrm{z} 620.2643$, found $\mathrm{m} / \mathrm{z} 620.2660$.

Supporting Information: Supporting Information contains copies of ${ }^{1} \mathrm{H}$ NMR and ${ }^{13} \mathrm{C}$ NMR spectra. 


\section{Acknowledgments}

We wish to thank the Institut de Chimie des Substances Naturelles for financial support and a fellowship for C.M. and the Labex LERMIT for financial support and a fellowship for C. P.

[1] a) A. De Meijere, F. Diederich in Metal-Catalyzed Cross-Coupling Reactions, $2^{\text {nd }} e d$, Wiley-VCH, Weinheim, 2004, Vol. 1-2; b) J. Hassan, M. Sévignon, C. Gozzi, E. Schulz, M. Lemaire, Chem. Rev. 2002, 102, 1359-1470.

[2] For reviews and leading references, see: a) A. Suzuki, Angew. Chem. 2011, 123, 6854-6869; Angew. Chem. Int. Ed. 2011, 50, 6722-6737; b) A. Suzuki, Y. Yamamoto, Chem. Lett. 2011, 40, 894-901; c) N. Miyaura, Bull. Chem. Soc. Jpn. 2008, 81, 1535-1553; d) N. Miyaura Top. Curr. Chem. 2002, 219, 11-59; e) F. Bellina, A. Carpita, R. Rossi, Synthesis 2004, 2419-2440; f) A. Suzuki, J. Organomet. Chem. 1999, 576, 147-168; g) N. Miyaura, A. Suzuki, Chem. Rev. 1995, 95, $2457-$ 2483; h) N. Miyaura, K. Yamada, A. Suzuki, Tetrahedron Lett. 1979, $20,3437-3440$

[3] a) For a highlight on this topic, see: M. Tobisu, N. Chatani, Angew. Chem. 2011, 121, 3617-3620; Angew. Chem. Int. Ed. 2009, 48, 35653568 ; b) for a recent example concerning the triple functionalization of phloroglucinol derivatives to yield tetraaryles, see: F. Zhao, Y.-F. Zhang, J. Wen, D.-G. Yu, J.-B. Wei, Z. Xi, Z.-J. Shi, Org. Lett. 2013 , $15,3230-3233$

[4] a) Z. Hassan, T. Patonay, P. Langer, Synlett 2013, 412-423; b) R. Rossi, F. Bellina, M. Lessi, Adv. Synth. Catal. 2012, 354, 1181-1255; c) R. Rossi, F. Bellina, M. Lessi, Tetrahedron 2011, 67, 6969-7025; d) R. Wang, K. Manabe, Synthesis 2009, 1405-1427; e) S. Schröter, C. Stock, T. Bach, Tetrahedron 2005, 61, 2245-2267.

[5] Y. Uozumi, M. Kikuchi, Synlett 2005, 1775-1778.

[6] a) D. J. Sinclair, M. S. Sherburn, J. Org. Chem. 2005, 70, 3730-3733; b) for mono-coupling using an excess of the dibromo arene, see also: E. Guillén, J. Hierrezuelo, R. Martínez-Mallorquín, J. M. LópezRomero, R. Rico, Tetrahedron 2011, 67, 2555-2561; c) it also has been shown that 1,8-dibromonaphthalene tends to favor dicoupling over mono-coupling: C. F. R. A. C. Lima, J. E. Rodriguez-Borges, L. M. N. B. F. Santos, Tetrahedron 2011, 67, 689-697.

[7] C.-G. Dong, T.-P. Liu, Q.-S. Hu, Synlett 2009, 1081-1086.

[8] E. Ullah, J. McNulty, A. Robertson, Eur. J. Org. Chem. 2012, 2127 2131.

[9] a) Tetrachloropyrimidine: M. Hussain, N. T. Hung, R. A. Khera, I. Malik, D. S. Zinad, P. Langer, Adv. Synth. Catal. 2010, 352, 14291433; b) pentachloropyridine: P. Ehlers, S. Reimann, S. Erfle, A. Villinger, P. Langer, Synlett 2010, 1528-1532; c) tetrabromothiophenes: D. T. Tùng, D. T. Tuấn, N. Rasool, A. Villinger, H. Reinke, C. Fischer, P. Langer, Adv. Synth. Catal. 2009, 351, 15951609; d) tetrabromopyrroles: T. T. Dang, R. Ahmad, T. T. Dang, H. Reinke, C. Fischer, P. Langer, Tetrahedron Lett. 2008, 49, 1698-1700.

[10] F. Beaumard, P. Dauban, R. H. Dodd, Synlett. 2010, 4033-4042.

[11] F. Beaumard, P. Dauban, R. H. Dodd, Org. Lett. 2009, 11, 1801-1804.

[12] a) F. Plisson, X.-C. Huang, H. Zhang, Z. Khalil, R. J. Capon, Chem. Asian J. 2012, 7, 1616-1623; b) H. Kamiyama, Y. Kubo, H. Sato, N. Yamamoto, T. Fukuda, F. Ishibashi, M. Iwao, Bioorg. Med. Chem 2011, 19, 7541-7550; c) H. Fan, J. Peng, M. T. Hamann, J.-F. Hi, Chem. Rev. 2008, 108, 264-287; d) A. R. Quesada, M. D. Garcia Gravalos, J. L. Fernandez Puentes, Br. J. Cancer 1996, 74, 677-682.

[13] a) P. Y. Zhang, I. L. K. Wong, C. S. W. Yan, X. Y. Zhang, T. Jiang, L. M. C. Chow, S. B. Wan, J. Med. Chem. 2010, 53, 5108-5120; b) H. Kang, W. Fenical, J Org. Chem. 1997, 62, 3254-3262.

[14] For implementation of this strategy towards such natural products, see: a) N. Fujikawa, T. Ohta, T. Yamaguchi, T. Fukuda, F. Ishibashi, M. Iwao, Tetrahedron 2006, 62, 594-604; b) M. Iwao, T. Takeushi, N. Fujikawa, T. Fukuda, F. Ishibashi, Tetrahedron Lett. 2003, 44, 4443-4446.
[15] For detailed mechanistic studies on the Suzuki-Miyaura crosscoupling, see: a) C. Amatore, A. Jutand, G. Le Duc, Chem. Eur. J. 2012, 18, 6616-6625; b) C. Amatore, A. Jutand, G. Le Duc, Angew. Chem. 2012, 124, 1408-1411; Angew. Chem. Int. Ed. 2012, 51, 1379 1382; c) C. Amatore, A. Jutand, G. Le Duc, Eur. Chem. J. 2011, 17 2492-2503; d) B. P. Carrow, J. F. Hartwig, J. Am. Chem. Soc. 2011 133, 2116-2119; e) C. Adamo, C. Amatore, I. Ciofini, A. Jutand, H. Lakmini, J. Am. Chem. Soc. 2006, 128, 6829-6836.

[16] Indeed recent studies have shown that the nature of the boron derivative can have an important role on the outcome of the reaction, mainly by influencing the rate of release of the active boronic acid see: a) A. J. J. Lennox, G. C. Lloyd-Jones, J. Am. Chem. Soc. 2012 134, 7431-7441; b) N. Zhang, D. J. Hoffman, N. Gutsche, J. Gupta, V. Percec, J. Org. Chem. 2012, 77, 5956-5964; c) M. Butter, J. N. Harvey, J. Jover, A. J. J. Lennox, G. C. Lloyd-Jones, P. M. Murray, Angew. Chem. 2010, 122, 5282-5286; Angew. Chem. Int. Ed. 2010, 49, 5156-5160; d) A. J. J. Lennox, G. C. Lloyd-Jones, Isr. J. Chem. 2010, 50, 664-674; e) R. Ting, C. W. Harwig, J. Lo, Y. Li, M. J. Adam, T. J. Ruth, D. M. Perrin, J. Org. Chem. 2008, 73, 4662-4670.

[17] These conditions were adapted from our previous work on one-pot double Suzuki-Miyaura couplings (see ref 11), for which we were able to lower the amount of catalyst and additive $(\mathrm{KCl})$ without hampering the efficiency of the reaction. Moreover, for a mono-coupling only 4 equivalents of base are necessary but, at this stage, we felt that it would be interesting to set reaction conditions that would already be suitable for the one-pot sequential double coupling reaction developed thereafter. The positive role of alkali halides additives such as $\mathrm{KCl}$ in Suzuki-Miyaura cross-couplings has been reported and rationalized early on for reactions involving aryltriflates : a) T. Oh-e, N. Miyaura, A. Suzuki, Synlett. 1990, 221-223; b) T. Oh-e, N. Miyaura, A. Suzuki, J. Org. Chem. 1993, 58, 2201-2208. Similar positive effects have also been witnessed for arylhalides, for a recent report on this subject, see: c) B. Zhang, J. Song, H. Liu, J. Shi, J.Ma, H. Fan, W. Wang, P. Zhang, B. Han, Green Chem. DOI: 10.1039/c3gc42088d.

[18] It has also been proposed that the $\mathrm{Pd} /$ phosphine ratio as well as the nature of the phosphine might also influence the diffusion process of the active catalyst and that increasing the diffusion would allow the active catalyst to "escape" the vicinity of the mono coupled adduct, thus favoring the selectivity of the process, see: ref 7 and C.-G. Dong, Q.-S. Hu, J. Am. Chem. Soc. 2005, 127, 10006-10007.

[19] For reviews on potassium trifluoroborates, see: a) S. Darses, J.-P. Genêt, Chem. Rev. 2008, 108, 288-325; b) G. A. Molander, N. Ellis Acc. Chem. Res. 2007, 40, 275-286. A notable advantage of using potassium trifluoroborates instead of boronic acids is that they help minimize and/or suppress unwanted side reactions such as protodeboronation and oxidative homocoupling; for more details see ref $16 \mathrm{c}$ and $16 \mathrm{~d}$

[20] For seminal work on one-pot double Suzuki-Miyaura couplings on unsymmetrical dihaloarenes, see: a) S. C. Anderson, S. T. Handy, Synthesis 2010, 2721-2724; b) S. Varello, S. T. Handy, Synthesis 2009, 138-142; c) S. T. Handy, T. Wilson, A. Muth, J. Org. Chem. 2007, 72, 8496-8500. For a one-pot double Suzuki-Miyaura coupling on differentiated diboron compounds, see: d) G. A. Molander, D. L. Sandrock J. Am. Chem. Soc. 2008, 130, 15792-15793.

[21] The corresponding $p$-methoxy terphenyl has been prepared using different procedures from non-commercial starting materials and in both cases the yields are under 50\%: a) M.-Y. Chang, C.-K. Chan, S.Y. Lin, M.-H. Wu Tetrahedron 2013, 69, 9616-9624; b) P. GarcíaGarcía, M. A. Fernández-Rodríguez, E. Aguilar Angew. Chem. 2009 121, 5642-5645; Angew. Chem. Int. Ed. 2009, 48, 5534-5537.

[22] T. Ishiyama, M. Murata, N. Miyaura, J. Org. Chem. 1995, 60, 75087510

Received: ((will be filled in by the editorial staff)) Published online: ((will be filled in by the editorial staff)) 


\section{Entry for the Table of Contents}

\section{Layout 2:}
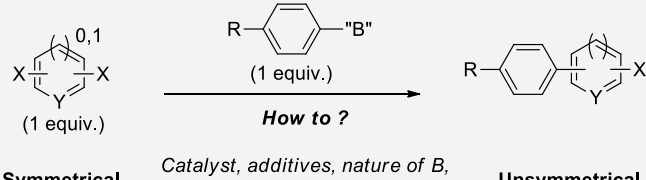

Symmetrical Catalyst, additives, nature of $B$

Unsymmetrical

38 cases studied

By studying several key parameters, the factors that allow achieving selective Suzuki-Miyaura mono-coupling on symmetrical dihaloarenes have been outlined.
This study ugeq?a? yigks the basis for the efficient synthesis of unsymmetrical triaryls from symmetrical dibromoarenes and arylditriflates using a one-pot desymmetrizing double Suzuki-Miyaura coupling.
Corinne Minard, Carole Palacio, Kevin Cariou, Robert H. Dodd*

Selective Suzuki-Miyaura MonoCouplings with Symmetrical Dibromoarenes and Arylditriflates for One-pot Synthesis of Unsymmetrical Triaryls.

Keywords: Suzuki-Miyaura, selective coupling, potassium trifluoroborates, triaryls 
